\title{
A MEASUREMENT OF THE KINETIC SUNYAEV-ZEL'DOVICH SIGNAL TOWARD MACS J0717.5+3745
}

\author{
J. SAYers ${ }^{1,11}$, T. MroczKowski ${ }^{1,2,12}$, M. Zemcov ${ }^{1,2}$, P. M. Korngut ${ }^{2}$, J. Bock $^{1,2}$, E. Bulbul ${ }^{3}$, N. G. CZAKON $^{1}$, E. Egami $^{4}$ \\ S. R. Golwala ${ }^{1}$, P. M. Koch ${ }^{5}$, K.-Y. Lin ${ }^{5}$, A. Mantz ${ }^{6}$, S. M. Molnar ${ }^{7}$, L. Moustakas ${ }^{2}$, E. PierPaOli $^{8}$, T. D. Rawle ${ }^{4}$,

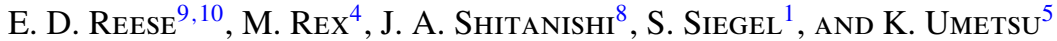 \\ ${ }^{1}$ Division of Physics, Math, and Astronomy, California Institute of Technology, 1200 East California \\ Boulevard, Pasadena, CA 91125, USA; jack@caltech.edu \\ 2 Jet Propulsion Laboratory, 4800 Oak Grove Drive, Pasadena, CA 91109, USA \\ ${ }^{3}$ Harvard-Smithsonian Center for Astrophysics, 60 Garden Street, Cambridge, MA 02138, USA \\ ${ }^{4}$ Steward Observatory, University of Arizona, 933 North Cherry Avenue, Tucson, AZ 85721, USA \\ ${ }^{5}$ Institute of Astronomy and Astrophysics, Academia Sinica, P.O. Box 23-141, Taipei 10617, Taiwan \\ ${ }^{6}$ Kavli Institute for Cosmological Physics, University of Chicago, 5640 South Ellis Avenue, Chicago, IL 60637, USA \\ ${ }^{7}$ LeCosPA Center, National Taiwan University, Taipei 10617, Taiwan \\ ${ }^{8}$ Department of Physics and Astronomy, University of Southern California, 3620 McClintock Avenue, Los Angeles, CA 90089, USA \\ ${ }^{9}$ Department of Physics and Astronomy, University of Pennsylvania, 209 South 33rd Street, Philadelphia, PA 19104, USA \\ ${ }^{10}$ Department of Physics, Astronomy, and Engineering, Moorpark College, 7075 Campus Road, Moorpark, CA 93021, USA \\ Received 2013 July 12; accepted 2013 September 25; published 2013 November 1
}

\begin{abstract}
We report our analysis of MACS J0717.5+3745 using 140 and $268 \mathrm{GHz}$ Bolocam data collected at the Caltech Submillimeter Observatory. We detect extended Sunyaev-Zel'dovich (SZ) effect signal at high significance in both Bolocam bands, and we employ Herschel-SPIRE observations to subtract the signal from dusty background galaxies in the $268 \mathrm{GHz}$ data. We constrain the two-band SZ surface brightness toward two of the sub-clusters of MACS J0717.5+3745: the main sub-cluster (named C), and a sub-cluster identified in spectroscopic optical data to have a line-of-sight velocity of $+3200 \mathrm{~km} \mathrm{~s}^{-1}$ (named B). We determine the surface brightness in two separate ways: via fits of parametric models and via direct integration of the images. For both sub-clusters, we find consistent surface brightnesses from both analysis methods. We constrain spectral templates consisting of relativistically corrected thermal and kinetic SZ signals, using a jointly-derived electron temperature from Chandra and XMMNewton under the assumption that each sub-cluster is isothermal. The data show no evidence for a kinetic SZ signal toward sub-cluster C, but they do indicate a significant kinetic SZ signal toward sub-cluster B. The model-derived surface brightnesses for sub-cluster B yield a best-fit, line-of-sight velocity of $v_{z}=+3450 \pm 900 \mathrm{~km} \mathrm{~s}^{-1}$, with $\left(1-\operatorname{Prob}\left[v_{z} \geqslant 0\right]\right)=1.3 \times 10^{-5}(4.2 \sigma$ away from 0 for a Gaussian distribution). The directly integrated sub-cluster B SZ surface brightnesses provide a best-fit $v_{z}=+2550 \pm 1050 \mathrm{~km} \mathrm{~s}^{-1}$, with $\left(1-\operatorname{Prob}\left[v_{z} \geqslant 0\right]\right)=2.2 \times 10^{-3}$ $(2.9 \sigma)$.
\end{abstract}

Key words: galaxies: clusters: individual (MACS J0717.5+3745) - galaxies: clusters: intracluster medium

Online-only material: color figures

\section{INTRODUCTION}

Measurements of large-scale peculiar velocities provide a direct probe of cosmological models and can be used to place constraints on parameters that are highly degenerate and/or unconstrained via other cosmological probes, such as measurements of primary cosmic microwave background (CMB) fluctuations (Bennett et al. 2013; Hinshaw et al. 2013; Planck Collaboration et al. 2013b, 2013c) and supernovae distance measurements (Conley et al. 2011; Suzuki et al. 2012). Specifically, these peculiar velocities depend on the properties and distributions of largescale structure, along with the characteristics of dark energy and the behavior of gravity on the corresponding length scales. Consequently, peculiar velocity measurements for large numbers of objects can probe the redshift evolution of the properties of dark energy (Bhattacharya \& Kosowsky 2008) and also distinguish between dark energy and modified gravity models (Kosowsky \& Bhattacharya 2009). In addition, measurements of an extremely large peculiar velocity for a single object (e.g., 1E0657-56, also known as the bullet cluster) can be used to directly test the validity of standard cosmological models (Hayashi \& White 2006; Lee \& Komatsu 2010; Thompson \& Nagamine 2012).

\footnotetext{
${ }^{11}$ Norris Foundation CCAT Postdoctoral Fellow.

${ }^{12}$ NASA Einstein Postdoctoral Fellow.
}

In the local universe, line-of-sight peculiar velocities can be measured using a combination of spectroscopy and distance measurements via the extragalactic distance ladder, generally using the relation described by Tully \& Fisher (1977). Such measurements have been used to constrain cosmological parameters like the total matter density $\Omega_{m}$ and the normalization of density fluctuations $\sigma_{8}$, generally finding good agreement with other cosmological probes (e.g., Feldman et al. 2010; Nusser \& Davis 2011; Ma et al. 2012). Unfortunately, uncertainties in the extragalactic distance ladder are proportional to distance, therefore preventing the application of these methods outside the local universe. In contrast, the kinetic Sunyaev-Zel'dovich (SZ) effect provides a direct measurement of the line-of-sight peculiar velocity of the distribution of hot electrons within galaxy clusters (Sunyaev \& Zel'dovich 1972; see Section 2). In addition, the surface brightness of the kinetic SZ signal is independent of redshift, depending only on the electron optical depth and line-of-sight peculiar velocity. Consequently, many groups have performed detailed studies of the cosmological constraints that would be possible with large-scale peculiar velocity surveys using the kinetic SZ signal (e.g., Bhattacharya \& Kosowsky 2008; Kosowsky \& Bhattacharya 2009; Mak et al. 2011).

Despite the great promise of kinetic SZ surveys, measurements of the kinetic SZ signal have proven to be a significant observational challenge. Over the past two decades, several 
attempts have been made to detect the kinetic SZ signal toward a variety of individual massive clusters. These observational efforts have used a range of instrumentation, including: the dedicated multi-band photometer SuZIE and its successors (Holzapfel et al. 1997; Benson et al. 2003), multiband data collected from a range of facilities (Kitayama et al. 2004), the moderate resolution spectroscopic receiver Z-Spec (Zemcov et al. 2012), and the two-band photometric imaging camera Bolocam (Mauskopf et al. 2012; Mroczkowski et al. 2012). None of these observations have made a highsignificance detection of the kinetic SZ signal, and the derived uncertainties on the line-of-sight peculiar velocities have not improved significantly from the first measurements with SuZIE, at least in part because none of the subsequent measurements have used instrumentation specifically designed to detect the kinetic SZ signal.

Recently, data from the Wilkinson Microwave Anisotropy Probe (WMAP) and Planck satellites have been used to place upper limits on bulk flows and rms variations in peculiar velocities via the kinetic SZ signal (Osborne et al. 2011; Planck Collaboration et al. 2013d). In addition, Hand et al. (2012) used a combination of Atacama Cosmology Telescope (ACT) and Sloan Digital Sky Survey III data to constrain the mean pairwise momentum of clusters using a kinetic SZ signature that is inconsistent with noise at a confidence level of $99.8 \%$. Furthermore, upper limits on the kinetic SZ power spectrum measured by the South Pole Telescope (SPT) have been used to inform cosmological simulations and to place constraints on the reionization history of the universe (Reichardt et al. 2012; Zahn et al. 2012).

One of the strongest hints of a kinetic SZ detection was presented in Mroczkowski et al. (2012, hereafter M12) toward the massive cluster MACS J0717.5+3745 using Bolocam measurements at 140 and $268 \mathrm{GHz}$. Motivated by this result, we have collected a significant amount of additional $268 \mathrm{GHz}$ Bolocam data toward this cluster. The results we obtain using this additional, deeper data are presented in this manuscript, which is organized as follows. In Section 2 we present the SZ effect and in Section 3 we describe previous analyses of MACS J0717.5+3745. In Section 4 we provide the details of our data reduction. We describe our model of the SZ signal toward MACS J0717.5+3745 in Section 5 and provide the corresponding constraints on the two-band SZ surface brightnesses of the cluster in Sections 6 and 7. In Section 8 we give the line-of-sight peculiar velocity constraints derived from these surface brightnesses, in Section 9 we put these results in a broader context, and in Section 10 we briefly summarize our analysis. We also include an Appendix, which fully details our treatment of the cosmic infrared background (CIB) in the $268 \mathrm{GHz}$ data.

\section{THE SZ EFFECT}

When a massive galaxy cluster is moving with respect to the rest frame of the $\mathrm{CMB}$, the Doppler-induced spectral distortion of the CMB due to the bulk motion of the electrons in the intracluster medium (ICM) is described by the kinetic SZ effect (e.g., Sunyaev \& Zel'dovich 1972; Birkinshaw 1999; Carlstrom et al. 2002). The change in CMB temperature due to the kinetic $\mathrm{SZ}$ effect is given by

$$
\frac{\Delta T_{\mathrm{CMB}}}{T_{\mathrm{CMB}}}=-\frac{v_{z}}{c} \tau_{e},
$$

where $v_{z}$ is the ICM peculiar velocity along the line-of-sight, $c$ is the speed of light, and $\tau_{e}$ is the total electron optical depth

$$
\tau_{e}=\int n_{e} \sigma_{T} d l
$$

for an electron density $n_{e}$ integrated along the line of sight $d l$ ( $\sigma_{T}$ is the Thompson cross section). We note that a positive peculiar velocity results in a negative temperature change, under the convention that a Doppler shift toward higher redshift corresponds to a positive value of $v_{z}$. In addition, we note that there are small relativistic corrections to the kinetic SZ signal (e.g., Nozawa et al. 1998a; Sazonov \& Sunyaev 1998; Nozawa et al. 2006; Chluba et al. 2012).

There is also a thermal SZ effect, which describes the Compton scattering of $\mathrm{CMB}$ photons off of high energy electrons in the ICM of massive clusters (e.g., Sunyaev \& Zel'dovich 1972; Rephaeli 1995a; Birkinshaw 1999; Carlstrom et al. 2002). Specifically, the change in CMB temperature due to the thermal SZ effect is given by

$$
\frac{\Delta T_{\mathrm{CMB}}}{T_{\mathrm{CMB}}}=f\left(v, T_{e}\right) y,
$$

where $f\left(v, T_{e}\right)$ encodes the frequency $v$ dependence, including relativistic corrections that depend on the electron temperature $T_{e}$ (e.g., Rephaeli 1995b; Itoh et al. 1998; Nozawa et al. 1998b; Itoh \& Nozawa 2004; Chluba et al. 2012) and

$$
y=\int n_{e} \sigma_{T} \frac{k_{B} T_{e}}{m_{e} c^{2}} d l
$$

( $k_{B}$ is Boltzmann's constant and $m_{e}$ is the electron mass). In the limit of an isothermal distribution, $y$ is directly and linearly proportional to the total electron optical depth $\tau_{e}$.

\section{PREVIOUS ANALYSES OF MACS J0717.5+3745}

MACS J0717.5+3745, located at $z=0.55$, was discovered as part of the Massive Cluster Survey (MACS; Ebeling et al. 2001, 2007) and is extremely massive and dynamically disturbed. As such, it has been the focus of many studies at a range of wavelengths, and it has been chosen as part of the six-cluster Hubble Space Telescope Frontier Fields program. ${ }^{13}$ Radio observations have shown that MACS J0717.5+3745 hosts the most powerful radio halo known (Edge et al. 2003; van Weeren et al. 2009; Bonafede et al. 2009), and strong lensing data have shown that MACS J0717.5+3745 has the largest known Einstein radius (Zitrin et al. 2009; Meneghetti et al. 2011; Waizmann et al. 2012). From both the galaxy distribution (Ebeling et al. 2004) and weak lensing studies (Jauzac et al. 2012; Medezinski et al. 2013), MACS J0717.5+3745 also appears to be part of a large, extended filamentary structure. In addition, it has the highest $\mathrm{X}$-ray temperature among all of the clusters in the MACS catalog (Ebeling et al. 2007).

Ma et al. (2009) performed a joint analysis using X-ray data, along with the measured galaxy positions and redshifts, and identified four distinct sub-clusters in MACS J0717.5+3745, from $\mathrm{N}$ to $\mathrm{S}$ labeled as $\mathrm{A}, \mathrm{B}, \mathrm{C}$, and $\mathrm{D}$ (see Figure 1). An independent strong lensing analysis described in Limousin et al. (2012) also identified four sub-clusters, with similar positions to the ones given in Ma et al. (2009). Both analyses found

\footnotetext{
13 http://www.stsci.edu/hst/campaigns/frontier-fields/
} 


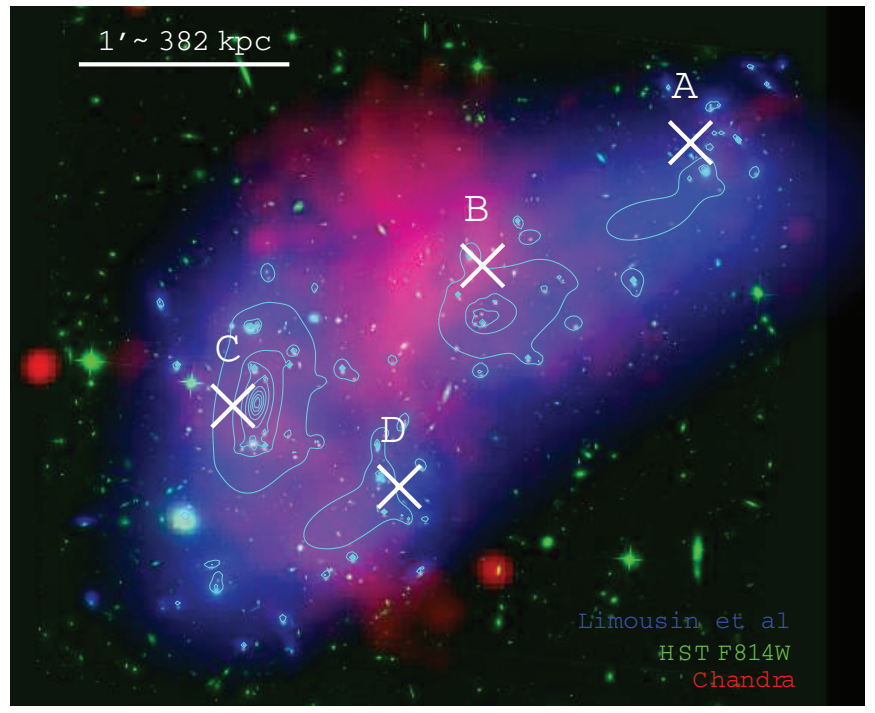

Figure 1. False-color composite image of MACS J0717.5+3745 with the lensing results of Limousin et al. (2012) in blue, the Hubble Space Telescope image using the F814W filter in green, and the Chandra X-ray image in red. The blue contours show the Limousin et al. (2012) result on a linear scale, and clearly indicate the four sub-clusters labeled A through $\mathrm{D}$, with white Xs marking the sub-cluster positions determined by Ma et al. (2009) from the galaxy distribution.

(A color version of this figure is available in the online journal.)

sub-cluster $\mathrm{C}$ to be the most massive system, and Ma et al. (2009) determined that sub-cluster $\mathrm{C}$ is probably the highly disturbed core of the main system. Sub-clusters B and D are assumed to be relatively intact cores of systems that are merging along a direction close to the line-of-sight. In particular, subcluster B is coincident with an X-ray temperature that is colder than the surrounding regions, indicating that its core has not been highly disrupted by the merger. From the spectroscopic data, Ma et al. (2009) found that sub-cluster B has a lineof-sight velocity that differs from the other components by approximately $3000 \mathrm{~km} \mathrm{~s}^{-1}$. Further indications of this large line-of-sight velocity for sub-cluster B were presented in M12, who found a similar best-fit velocity by using X-ray and SZ measurements to constrain the kinetic SZ signal toward that sub-cluster, although the statistical significance of their kinetic SZ constraint on the velocity is modest $(\simeq 2 \sigma)$. This wide range of observational data toward MACS J0717.5+3745 is therefore converging to what appears to be a coherent picture of this complex system.

\section{DATA REDUCTION}

\subsection{Bolocam}

We observed MACS J0717.5+3745 with Bolocam from the Caltech Submillimeter Observatory (CSO) for a total of $12.5 \mathrm{hr}$ at $140 \mathrm{GHz}$ and for a total of $27.3 \mathrm{hr}$ at $268 \mathrm{GHz}$, where the effective band centers are quoted for a CMB spectrum. Compared to the previous Bolocam analysis presented in M12, this represents an additional $19.3 \mathrm{hr}$ of data collected at $268 \mathrm{GHz}$ in 2012 December. In contrast to the original $8.0 \mathrm{hr}$ of $268 \mathrm{GHz}$ integration used in M12, much of which was collected in poor observing conditions with a $225 \mathrm{GHz}$ optical depth $\tau_{225}>0.10$, most of the additional $19.3 \mathrm{hr}$ of $268 \mathrm{GHz}$ integration was obtained with $\tau_{225} \simeq 0.05$. This additional data was therefore collected during the lowest opacity conditions generally available from the CSO.
The Bolocam instrument has an $8^{\prime}$ diameter circular field of view (FOV), and point-spread functions (PSFs) that are approximately Gaussian with full widths at half-maximums (FWHMs) equal to $58^{\prime \prime}$ and $31^{\prime \prime}$ at 140 and $268 \mathrm{GHz}$, respectively (Glenn et al. 2002; Haig et al. 2004). All of our Bolocam observations of MACS J0717.5+3745 involved scanning the CSO in a Lissajous pattern with an rms velocity of approximately $4^{\prime} \mathrm{s}^{-1}$. The details of our data reduction are given elsewhere (Sayers et al. 2011; M12), and we briefly summarize our procedure below.

First, we obtain pointing corrections accurate to $5^{\prime \prime}$ using frequent observations of nearby quasars, and obtain an absolute flux calibration accurate to $5 \%$ and $10 \%$ at 140 and $268 \mathrm{GHz}$, respectively, using observations of Uranus and Neptune (Griffin \& Orton 1993; Sayers et al. 2012). We note that Hasselfield et al. (2013) recently determined the brightness temperature of Uranus to be $106.7 \pm 2.2 \mathrm{~K}$ at $149 \mathrm{GHz}$ using ACT observations calibrated against the primary $\mathrm{CMB}$ anisotropies measured by the WMAP satellite. Also, Planck Collaboration et al. (2013a) recently determined the brightness temperature of Uranus to be $108.4 \pm 2.9 \mathrm{~K}$ at $143 \mathrm{GHz}$ based on Planck data. Our calibration model assumes a brightness temperature of $106.6 \pm 3.5 \mathrm{~K}$ for the $140 \mathrm{GHz}$ Bolocam bandpass, which was measured in Sayers et al. (2012) by extrapolating the WMAP $94 \mathrm{GHz}$ brightness measurements presented in Weiland et al. (2011) using the model of Griffin \& Orton (1993). This model predicts the brightness temperature of Uranus to increase with decreasing frequency. As a result, the ACT and Planck measurements imply a best-fit $140 \mathrm{GHz}$ brightness temperature that is approximately $2.5 \mathrm{~K}$ higher than our assumed value of $106.6 \mathrm{~K}$. However, this difference is comparable to the ACT and Planck measurement uncertainties, and it is well below our estimated 5\% flux calibration uncertainty at $140 \mathrm{GHz}$. We therefore have not updated our calibration model. Furthermore, we note that the accuracy of the ACT and Planck Uranus brightness temperatures is $2 \%-3 \%$, which is only slightly smaller than the $3.3 \%$ accuracy of our assumed $140 \mathrm{GHz}$ brightness temperature. Furthermore, our $140 \mathrm{GHz}$ calibration uncertainty receives an approximately equal contribution from our $3.1 \%$ beam solid angle uncertainty. Revising our flux calibration using ACT and Planck would thus not have a significant effect on our overall calibration uncertainty, which itself is already sub-dominant to measurement uncertainties (see Table 2). Finally, we note that our $10 \%$ flux calibration at $268 \mathrm{GHz}$ is limited largely by atmospheric fluctuations, and therefore a more accurate Uranus brightness temperature at that frequency would have no effect on our overall calibration uncertainty.

To remove atmospheric fluctuations from the data, we first subtract a template of the common mode signal over the FOV, and we then high-pass filter (HPF) the time-stream data at 250 and $500 \mathrm{mHz}$ at 140 and $268 \mathrm{GHz}$, respectively. The large amplitude of the atmospheric fluctuations in the $268 \mathrm{GHz}$ data necessitates this more aggressive HPF, and this filtering represents a slight change from the M12 analysis, which used a $250 \mathrm{mHz}$ HPF for both data sets. We used a scan speed of $\simeq 4^{\prime} \mathrm{s}^{-1}$ for our observations, and the HPFs at 250 or $500 \mathrm{mHz}$ therefore correspond to angular scales of $16^{\prime}$ and $8^{\prime}$, respectively. Consequently, the maximum angular scale preserved by our filtering is largely set by the common mode subtraction over Bolocam's 8' FOV. Because our processing removes astronomical signals with angular sizes larger than the $8^{\prime} \mathrm{FOV}$, we determine the map-space transfer function at each wavelength by reverse-mapping and processing an image 
template through the entire reduction pipeline. We estimate the instrumental and atmospheric noise in our images by forming 1000 separate jackknife realizations of the data, where a randomly selected subset of half the single observations is multiplied by -1 to remove all astronomical signals. There are 75 single 10 minute observations of MACS J0717.5+3745 at $140 \mathrm{GHz}$ and 164 single 10 minute observations of MACS $\mathrm{J} 0717.5+3745$ at $268 \mathrm{GHz}$.

To account for noise from unwanted astronomical signals, we first add a different random realization of the primary CMB fluctuations, using the power spectrum measurements from the SPT, to each jackknife (Reichardt et al. 2012; Story et al. 2012). At $140 \mathrm{GHz}$, we add an additional random realization of the CIB, again based on the measured SPT power spectra (Hall et al. 2010), under the assumption that the fluctuations are Gaussian. This assumption is not strictly true, but the CIB fluctuations are more than an order of magnitude dimmer than the other noise fluctuations in the data, and therefore a breakdown of this assumption is not likely to have a noticeable effect on our results. The CIB is significantly brighter at $268 \mathrm{GHz}$, and we therefore use a much more detailed model to account for it in those data, as described in the Appendix. Throughout this manuscript we refer to these 1000 jackknife plus astronomical noise realizations as "noise realizations."

For the analyses described in this manuscript, we make use of the Bolocam data in two different ways. We use images of the processed data, which cover a maximum size of $14^{\prime} \times 14^{\prime}$, to constrain parametric models of the astronomical signals (see Section 5). This analysis involves convolving the model with the signal transfer function of the data processing and the Bolocam PSF. To determine best-fit parameters for a given model, we use the generalized least squares fitting algorithm MPFITFUN (Markwardt 2009) under the simplifying assumption that the map noise covariance matrix is diagonal. We have demonstrated that this fitting method produces unbiased estimates of the bestfit parameter values (Sayers et al. 2011), although in some cases it does produce a slightly biased estimate of the uncertainties on these best-fit parameters. Therefore, to fully account for all the subtleties of our noise, we derive all the parameter uncertainties via the spread of best-fit values we obtain from applying the same fitting algorithm to a sample of 1000 noise plus signal realizations. Each noise plus signal realization is generated by adding a noise realization to the best-fit model found for the real data.

We also deconvolve the transfer function of the data processing to obtain unbiased images after first reducing the image to a maximum size of $10^{\prime} \times 10^{\prime}$ to prevent significant amplification of the noise on the largest angular scales. One subtlety in this process is the fact that the signal transfer function is equal to 0 at an angular wavenumber of 0 (i.e., the DC signal level of the image is unconstrained). We therefore use the parametric model fits to constrain the DC signal level, as described in Section 6.1. As a result, the deconvolved images have some model dependence. Consequently, to ensure that the uncertainties on the model accurately represent the underlying uncertainties on the data, both for the model fits alone and for the results derived from the deconvolved images, the model must provide an acceptable fit quality. By requiring a model with an acceptable fit quality, we ensure that the results derived from model fits will be consistent with those derived from the deconvolved images. To estimate the noise in the deconvolved images, we also deconvolve the transfer function from each of the 1000 noise realizations.

\subsection{Chandra}

Our analysis of the Chandra X-ray exposures of MACS $\mathrm{J} 0717.5+3745$ is nearly identical to the analysis described in M12, and we briefly summarize the main aspects below. As in M12, we utilize both Chandra ACIS-I X-ray observations of MACS J0717.5+3745 (ObsIDs 1655 and 4200), for a total exposure time of $81 \mathrm{ks}$ (see Reese et al. 2010 for the reduction details). From these X-ray data we compute pseudo-pressure

$$
P_{e}=n_{e} k_{B} T_{e} \simeq \sqrt{\frac{4 \pi(1+z)^{3} S_{X}}{l \Lambda_{e e}\left(T_{e}, Z\right)}} k_{B} T_{e},
$$

where $S_{X}$ is the X-ray surface brightness

$$
S_{X}=\frac{1}{4 \pi(1+z)^{3}} \int n_{e}^{2} \Lambda_{e e}\left(T_{e}, Z\right) d l,
$$

$l$ is the effective line-of-sight extent of the ICM, and $\Lambda_{e e}\left(T_{e}, Z\right)$ is the X-ray emissivity as a function of $T_{e}$ and metallicity $Z$. To generate pseudo-pressure maps from the Chandra images, we first bin the data using contbin (Sanders 2006). We construct the pseudo-pressure maps from $T_{e}$ maps generated by computing $T_{e}$ within each bin and $n_{e}$ maps computed from the X-ray surface brightness (see Equation (5)). To rescale the pseudo-pressure maps to units of Compton- $y$, we need to determine the value of $l$ (which in M12 was done using $31 \mathrm{GHz}$ Sunyaev-Zel'dovich array (SZA) data, but in practice is left as a free parameter in all of our fits). This X-ray template for the thermal SZ signal, which is simply a rescaling of the X-ray pseudo-pressure map, is called a "pseudo-Compton- $y$ map" throughout this manuscript. For consistency with M12, we employ the same pseudo-Compton-y map that was generated for that analysis. We note that this map was generated using CIAO version 4.3 and calibration database (CALDB) version 4.4.5 (Fruscione et al. 2006).

New for this analysis compared to M12, we also generate 20 realizations of the pseudo-Compton- $y$ map that are fluctuated by the X-ray measurement uncertainties on $T_{e}$, which dominate the uncertainty of the pseudo-pressure maps (see Figure 2). We note that, in addition to measurement uncertainties, the pseudoCompton- $y$ maps are also subject to possible systematic errors due to gas clumping within the ICM. Clumping is defined as

$$
C=\frac{\left\langle n_{e}^{2}\right\rangle}{\left\langle n_{e}\right\rangle^{2}}
$$

and from the equations listed above, we see that the pseudoCompton- $y$ maps are sensitive to $\sqrt{C}$. Typical clumping factors of $C \simeq 1.1-1.2$ within $R_{500}$ are expected from simulations (e.g., Zhuravleva et al. 2013). Assuming clumping is uncorrelated with temperature variations, clumping is sub-dominant to the variations included in the input temperature maps, and we therefore do not include any additional uncertainty from clumping in our 20 realizations of the pseudo-Compton- $y$ maps. In addition, we note that the average systematic trend for X-ray surface brightness to be boosted by clumping is mitigated by the fact that the amplitude of the Compton- $y$ maps is constrained by the SZ data via the factor of $l$.

We also use the Chandra data to constrain the electron temperature $T_{e}$ within two $1^{\prime}$ diameter regions centered on subclusters B and C (these temperatures are applied to our analysis in Section 8). In contrast to the pseudo-Compton- $y$ maps, which we obtain via the same reduction that was used in M12, we 


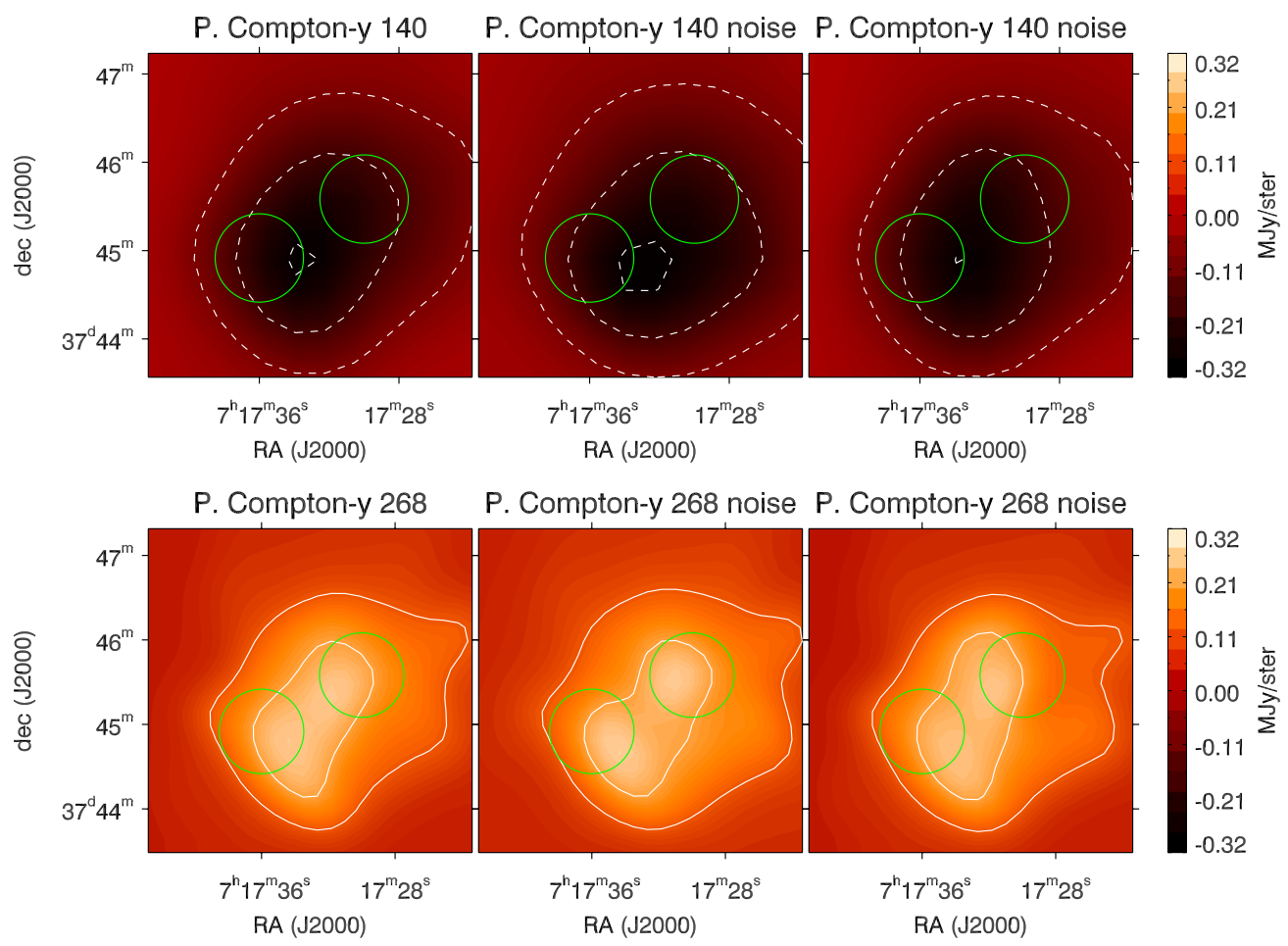

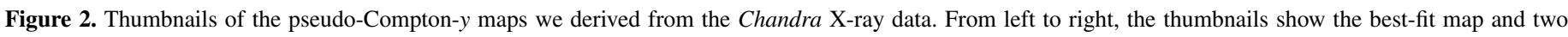

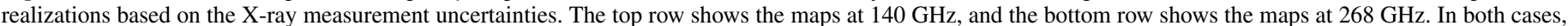

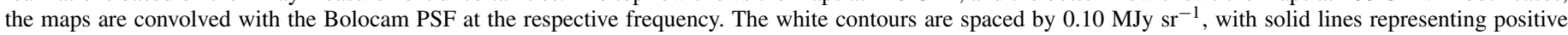

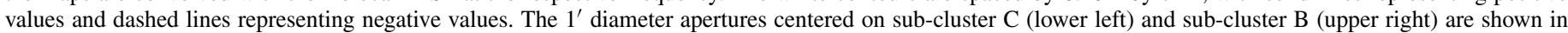
green. Note that the approximate conversion factor from $\mathrm{MJy} \mathrm{sr}^{-1}$ to $y$ is $-1 \times 10^{-3}$ at $140 \mathrm{GHz}$ and $+1 \times 10^{-3}$ at $268 \mathrm{GHz}$.

(A color version of this figure is available in the online journal.)

constrain these values of $T_{e}$ using maps generated with CIAO version 4.5 and calibration database (CALDB) version 4.5.6 (Fruscione et al. 2006). We fit the temperatures and metallicities of the regions in XSPEC (Dorman \& Arnaud 2001) using the Astrophysical Plasma Emission Code (APEC) model (Smith et al. 2001). We find $T_{e}=13.8_{-1.3}^{+1.6} \mathrm{keV}$ for sub-cluster $\mathrm{B}$ and $T_{e}=24.4_{-3.8}^{+7.8} \mathrm{keV}$ for sub-cluster $\mathrm{C}$, using the extended C-statistic to determine the temperature likelihoods. ${ }^{14}$

\subsection{XMM-Newton}

To better constrain the electron temperatures of sub-clusters $\mathrm{B}$ and $\mathrm{C}$, we make use of $\simeq 200 \mathrm{ks}$ of XMM-Newton X-ray data toward MACS J0717.5+3745. These data became public in 2012 October (ObsIDs; 0672420101,0672420301, 0672420301), and therefore were not included in M12. We perform the $X M M$ MOS data processing and background modeling with the $X M M$ Extended Source Analysis Software using the methods reported in Kuntz \& Snowden (2008) and Snowden et al. (2008). The details of our XMM analysis are described fully in Bulbul et al. (2012), and we provide a summary and discuss important differences here.

Our XMM-Newton data analysis includes the production of the calibrated event files, filtering for the high intensity soft proton flares, and the determination of the background intensity in each observation. The net exposure time after filtering the event files for good time intervals is $155 \mathrm{ks}$. Given the superior

\footnotetext{
14 Our uncertainties on the Chandra-derived temperature of sub-cluster B are significantly lower than the values reported in Table 3 of M12. This is due to the fact that our current analysis uses a $1^{\prime}$ diameter region, while the M12 analysis used a $40^{\prime \prime}$ diameter region (both analyses use a $1^{\prime}$ diameter region for sub-cluster $\mathrm{C}$ ). There are additional $<5 \%$ differences due to the updated calibration we use for our current analysis.
}

spatial resolution of Chandra, we use both Chandra and XMM to identify regions contaminated by extragalactic X-ray sources not associated with the cluster gas. Excluding these regions, we extract spectra using $1^{\prime}$ diameter regions centered on subclusters $\mathrm{B}$ and $\mathrm{C}$, identical to the regions we use for the Chandra analysis. The temperature gradient is not large, and so contamination by adjacent regions (e.g., other sub-clusters) due to the wider PSF of $X M M$ should not affect the extracted temperature for each region.

For each extracted spectrum, we model a superposition of three main background components: quiescent particle background, soft X-ray background emission (including solar wind charge exchange, Galactic halo, local hot bubble, and extragalactic unresolved sources), and residual contamination from soft protons (Kuntz \& Snowden 2008). As in Snowden et al. (2008), we model the contamination due to unresolved point sources using an absorbed power law component with spectral index $\alpha=1.46$ and normalization $=8.88 \times 10^{7}$ photons $\mathrm{keV}^{-1} \mathrm{~cm}^{-2} \mathrm{~s}^{-1}$ at $1 \mathrm{keV}$.

We simultaneously fit all of the EPIC-MOS spectra using the energy range $0.3-10.0 \mathrm{keV}$. As with the Chandra spectral analysis, we use the absorbed APEC model to fit the cluster emission, employing the extended C-statistic for our likelihood analysis within each sub-cluster region.

From the $X M M$ data, we find $T_{e}=10.8_{-0.5}^{+0.5} \mathrm{keV}$ for subcluster B and $T_{e}=18.6_{-1.4}^{+1.6} \mathrm{keV}$ for sub-cluster $\mathrm{C}$. We note that these values are $\simeq 25 \%$ lower than the electron temperatures we derive from the Chandra data. This systematic difference at high temperature is consistent with previous comparisons between $X M M$ and Chandra (e.g., Nevalainen et al. 2010; Li et al. 2012; Mahdavi et al. 2013), although we note that, in our case, the statistical significance of the difference is relatively small 
$(\lesssim 2 \sigma)$. We consequently choose to combine the temperature measurements from the two X-ray observatories, and we obtain maximum likelihood values of $T_{e}=11.4_{-0.5}^{+0.5} \mathrm{keV}$ for subcluster B and $T_{e}=19.9_{-1.4}^{+1.5} \mathrm{keV}$ for sub-cluster $\mathrm{C}$. We explore the impact of using this joint temperature constraint, rather than the constraint from either $X M M$ or Chandra individually, in Section 9.2.

\section{MODEL OF THE SZ SIGNAL}

In order to model the SZ signals from the ICM of MACS J0717.5+3745, we employ the pseudo-Compton- $y$ map to describe the overall shape of the thermal SZ signal. The $\mathrm{X}$-ray data we use to create the pseudo-Compton- $y$ map depend negligibly on the line-of-sight velocity of the ICM, and the map therefore provides a spatial template for the thermal SZ signal that is free from contamination from the kinetic SZ signal. We then convert this map to units of surface brightness in each Bolocam observing band according to the thermal SZ equations (Sunyaev \& Zel'dovich 1972), including relativistic corrections (Itoh et al. 1998; Nozawa et al. 1998a, 1998b; Itoh \& Nozawa 2004). For this conversion we compute the responsivity-weighted average bandpass over all the Bolocam detectors, and from this spectrum we determine the effective band center for a thermal SZ spectrum. Due to relativistic corrections, this effective band center depends on the ICM temperature. For the $140 \mathrm{GHz}$ band, both the thermal and kinetic SZ band centers are $\simeq 140 \mathrm{GHz}$, while the $268 \mathrm{GHz}$ thermal SZ band center is $\simeq 275 \mathrm{GHz}$ and the kinetic SZ band center is $\simeq 268 \mathrm{GHz}$. Then, for each Bolocam band, we convolve the pseudo-Compton- $y$ map with both the Bolocam PSF and the transfer function of the data processing.

We first constrain the normalization of the pseudo-Compton- $y$ map via a simultaneous fit to both the 140 and $268 \mathrm{GHz}$ Bolocam data. Physically, this corresponds to a constraint on the effective line-of-sight extent of the ICM $l$, under the assumption of zero kinetic SZ signal. For this fit, we use only the data within a $4^{\prime} \times 4^{\prime}$ square region approximately centered on the peak of the $\mathrm{SZ}$ signal at $140 \mathrm{GHz}$. We choose this region because it is large enough to contain the bulk of the SZ signal and small enough to mitigate the effects of the large-angular-scale atmospheric noise in the $268 \mathrm{GHz}$ data. The quality of this fit is very poor, with $\chi^{2}=853.4$ for 717 degrees of freedom, indicating that the pseudo-Compton- $y$ map alone is inadequate to describe our Bolocam SZ data (see Figure 3).

Motivated by this poor fit, along with the significant differences in the line-of-sight velocities measured by Ma et al. (2009) for the four identified sub-clusters in MACS J0717.5+3745, and the results from M12, we consider additional components to our model of the ICM. To determine which, if any, additional model components are required in order to describe the data, we perform a simulated $F$-test according to the procedure described in N. G. Czakon (2013, in preparation). To perform this test we first insert the baseline model into each of our 1000 noise realizations (in this case the baseline model is the pseudo-Compton- $y$ map with our best-fit single normalization). We then fit two models to each of these realizations, one consisting of only the baseline model, and one with an extension to the baseline model. We compute the value of $\Delta \chi^{2}$ from these two separate fits for each of the 1000 realizations, and the resulting values provide a measurement of the distribution of $\Delta \chi^{2}$ for the null hypothesis that the model extension is not required by the data.
There are several possible model extensions to consider, and we therefore proceed according to the following decision tree: (1) determine the value of $\Delta \chi^{2}$ separately for each possible model extension; (2) perform the simulated $F$-test to determine which extension is most preferred by the data; (3) if the most preferred model extension is preferred at a high enough significance, which we quantify based on a probability to exceed (PTE) from the simulated $F$-test, then the model extension is added to the baseline model. These steps are repeated until none of the possible model extensions have a simulated $F$-test PTE below our threshold, which we have chosen to be equal to 0.02 . $^{15}$

As a first possible model extension, we consider a smooth template of the SZ signal centered on one of the four sub-clusters according to the positions given by Ma et al. (2009), allowing for different normalizations of the template at 140 and $268 \mathrm{GHz}$. We construct the SZ template according to the average profile constrained by Bolocam for a sample of 45 clusters (Sayers et al. 2013a), fixing the scale radius according to the estimated mass of each sub-cluster, which we obtain by using the ratios of sub-cluster masses determined by Limousin et al. (2012), in combination with the whole-cluster value of $M_{500}$ determined by Mantz et al. (2010). We note that this template represents a more physically motivated model than the Gaussian profile assumed by M12. We perform four separate fits, in each case fitting a single normalization to the pseudo-Compton-y map (i.e., assuming that the pseudo-Compton- $y$ map contains only thermal SZ signal), along with separate normalizations at 140 and $268 \mathrm{GHz}$ for an SZ template centered on one of the four sub-clusters (i.e., allowing the SZ template for that sub-cluster to be free to include any arbitrary mixture of thermal and kinetic SZ signal). We find values of $\Delta \chi^{2}$ equal to 48.3, 108.1, 9.5, and 26.3 when the model contains an additional SZ template coincident with sub-clusters A, B, C, and D, respectively. These values of $\Delta \chi^{2}$ correspond to $F$-test PTEs of $0.001,4 \times 10^{-7}$, 0.213 , and 0.026 , respectively. We note that the second value is extrapolated, due to the fact that we only have 1000 realizations of $\Delta \chi^{2}$.

As another possible extension to the baseline model of a pseudo-Compton-y map with a single normalization in both Bolocam bands, we also explore the option of allowing for different normalizations of the pseudo-Compton- $y$ map at 140 and $268 \mathrm{GHz}$. Physically, this would represent a single bulk velocity for the entire cluster, with the cluster being isothermal so that the kinetic SZ signal has a spatial profile identical to the spatial profile of the thermal SZ signal. This fit results in a value of $\Delta \chi^{2}=52.1$, which corresponds to a simulated $F$-test PTE of $3 \times 10^{-4}$, where we have again used an extrapolation due to our finite number of realizations. Therefore, according to our $F$-test decision tree, we assume a new baseline model consisting of the pseudo-Compton- $y$ with a single normalization, along with a SZ template centered on sub-cluster B, as this is the model extension with the smallest simulated $F$-test PTE.

To fully characterize the fit of this new baseline model, we insert the best-fit model into each of our 1000 noise realizations, and then fit the same model to each of these realizations. The best-fit model has an overall $\chi^{2}=745.3$ for 715 degrees of

\footnotetext{
15 As we describe below, we consider five independent potential model extensions, and consequently a possible total of $2^{5}=32$ model permutations. Our PTE threshold, which is necessarily somewhat arbitrary, is therefore small enough to ensure that a random fluctuation among this set of 32 permutations is unlikely to produce a PTE small enough for us to include an extension that is not justified by the data. We explore the sensitivity of our results to this PTE threshold in detail in Section 6.2.
} 

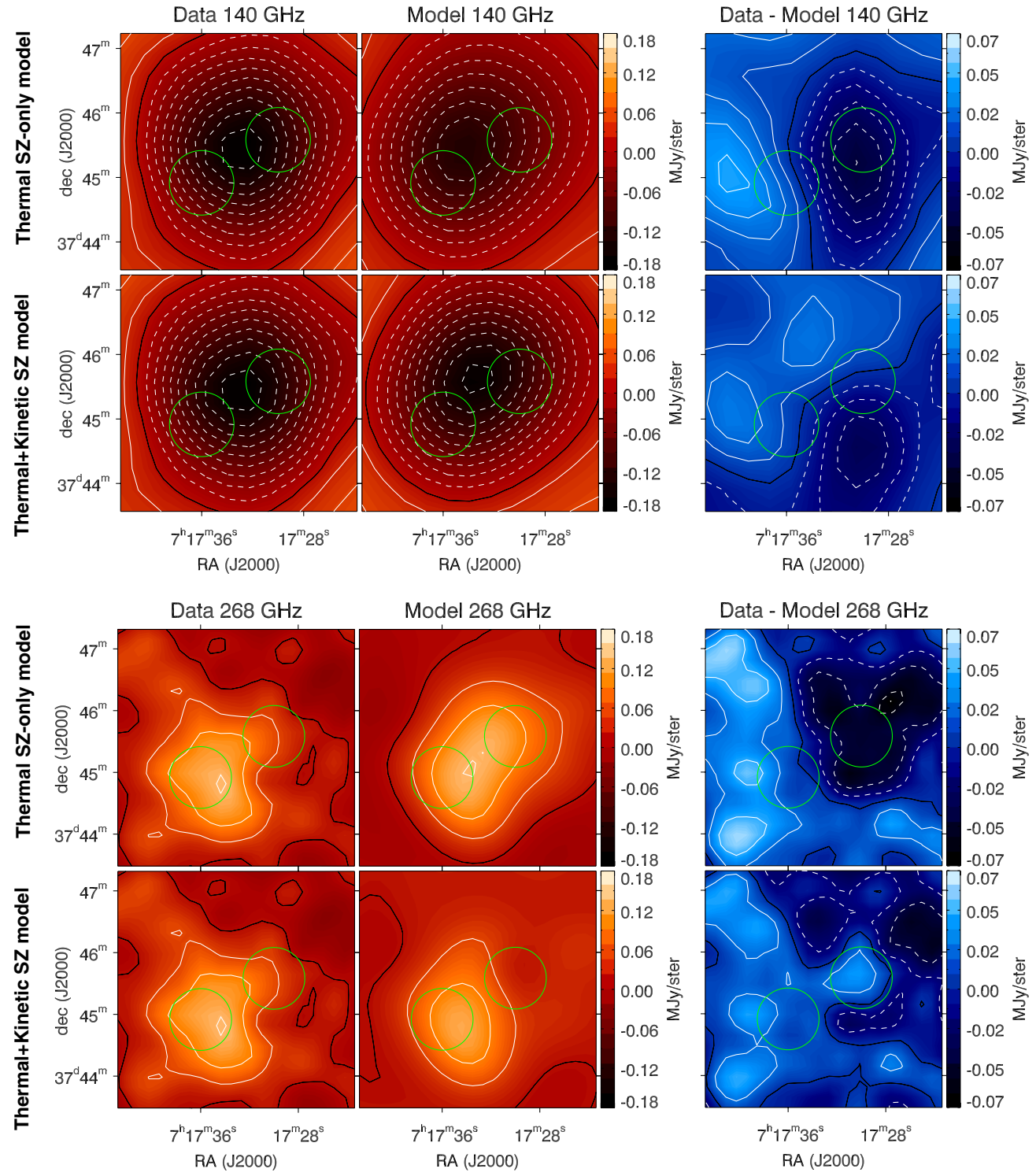

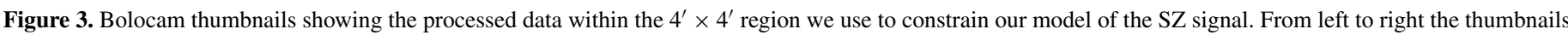

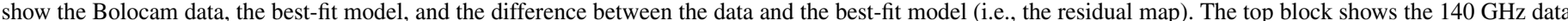

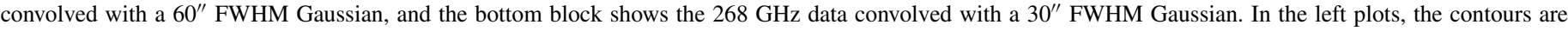

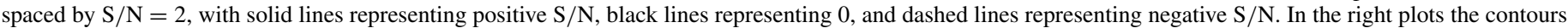

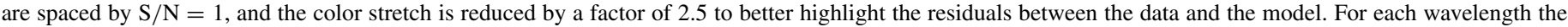

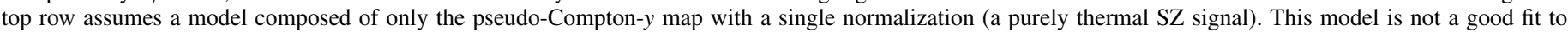

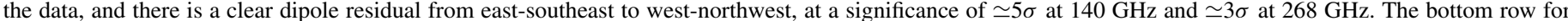

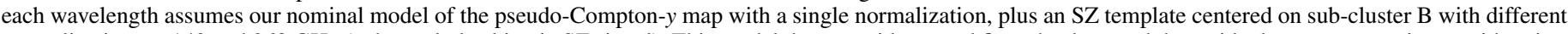

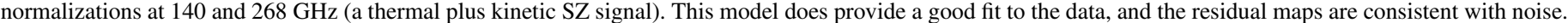
The green circles are centered on sub-cluster C (lower left) and sub-cluster B (upper right), with diameters of $60^{\prime \prime}$.

(A color version of this figure is available in the online journal.)

freedom, which corresponds to a PTE of 0.29 based on the fits to the noise realizations, indicating that it provides an adequate description of our Bolocam SZ data.

Using this new baseline model, we again follow the $F$-test decision tree to determine whether the data still require an additional model extension. We perform three new fits that introduce an additional SZ component at A, C, and D to our baseline model. For these three fits, the value of $\Delta \chi^{2}$ is 23.9, 8.4, and 21.1, which corresponds to a simulated $F$-test PTE of 0.033 , 0.260 , and 0.046 , respectively. As before, we also perform a fit allowing the normalization of the pseudo-Compton- $y$ map to be different at 140 and $268 \mathrm{GHz}$, and we find a $\Delta \chi^{2}=0.1$, with an associated simulated $F$-test PTE of 0.963 . All of these fits have
PTEs larger than 0.02, and we therefore conclude that none of these additional degrees of freedom are required to describe our data. Consequently, our baseline model of the $\mathrm{SZ}$ signal includes a pseudo-Compton- $y$ map with a single normalization, along with an SZ template centered on sub-cluster B with separate normalizations at 140 and $268 \mathrm{GHz}$ (see Figure 3).

The data's strong lack of a preference for separate normalizations of the pseudo-Compton- $y$ map at 140 and $268 \mathrm{GHz}$ justifies our choice of that model to describe the thermal component of the SZ signal. Furthermore, the best-fit normalization of the pseudo-Compton- $y$ map is $1.08 \pm 0.11$. The pseudo-Compton- $y$ map was normalized based on the integrated SZ signal measured at $31 \mathrm{GHz}$ by the SZA as reported in M12. Compared to 

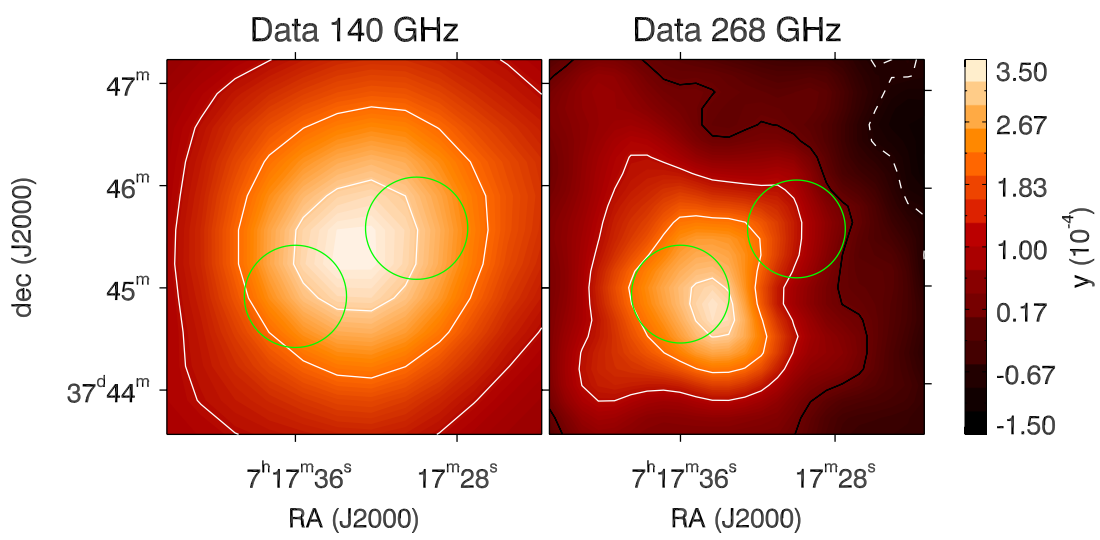

Figure 4. Thumbnails of the deconvolved Bolocam images at 140 and $268 \mathrm{GHz}$. We have scaled both images to units of Compton-y, including positionally dependent relativistic corrections based on the X-ray-determined temperature map. The relativistic corrections generally range from $8 \%$ to $15 \%$ at $140 \mathrm{GHz}$ and from $20 \%$ to $40 \%$ at $268 \mathrm{GHz}$. The $140 \mathrm{GHz}$ image is smoothed with a $60^{\prime \prime}$ Gaussian and the $268 \mathrm{GHz}$ image is smoothed with a $30^{\prime \prime}$ Gaussian. The contours are spaced by $1 \times 10^{-4}$, with solid lines showing positive $y$ and dashed lines showing negative $y$. The green circles show the $1^{\prime}$ diameter apertures centered on sub-cluster $\mathrm{C}$ (lower left) and sub-cluster B (upper right). The total Compton- $y$ signal toward sub-cluster C is nearly identical at the two wavelengths, whereas there is a clear difference toward sub-cluster B.

(A color version of this figure is available in the online journal.)

the Bolocam observing bands, the kinetic SZ signal is a factor of $\simeq 2$ dimmer compared to the thermal SZ signal in the SZA observing band. Therefore, the consistent normalizations of the pseudo-Compton-y map found by Bolocam and SZA further indicate that it provides a good description of the thermal SZ signal toward MACS J0717.5+3745. As an additional crosscheck, we also refit the normalization of the pseudo-Compton- $y$ map using the Bolocam data, but exclude the data within a $1^{\prime}$ diameter aperture that is centered on sub-cluster B. This fit, which did not include any additional SZ components, results in a best-fit normalization of $1.13 \pm 0.08$ for the pseudo-Compton$y$ map. The fit quality is good, with a PTE of 0.38 , indicating that, outside sub-cluster B, the M12 pseudo-Compton- $y$ map, which was normalized to SZA, describes the Bolocam data well. Although these results serve as additional evidence that our model choice is physically justified, we emphasize that our results (described below) do not strictly depend on the pseudoCompton- $y$ map being a good template for the thermal SZ signal, only that our model is physically motivated and provides an adequate description of the data.

\section{MEASUREMENT OF THE SZ SPECTRUM TOWARD SUB-CLUSTER B}

\subsection{Two-band SZ Photometry}

Based on the requirement of an additional model component centered on sub-cluster B to describe our data, we compute the SZ brightness at both 140 and $268 \mathrm{GHz}$ toward that sub-cluster. In order to eliminate as much contamination from other regions of the cluster as possible, we use a circular aperture with a diameter of $1^{\prime}$, which is slightly larger than the PSF FWHM at $140 \mathrm{GHz}$. We first compute the average surface brightnesses within this aperture using the best-fit model from Section 5, convolved with the Bolocam PSF to accurately represent the resolution of the measurement. To include all of the subtle effects of the noise, such as the correlations between pixels due to residual atmospheric noise and primary CMB fluctuations, we also compute the average surface brightness within the same aperture using the model fits to the 1000 noise realizations. Kolmogorov-Smirnov (KS) tests against Gaussians on the distributions of 1000 values at 140 and $268 \mathrm{GHz}$ yield PTEs of 0.19 and 0.93 , respectively, and therefore indicate that our noise is Gaussian within our ability to measure it. Using these model fits, we estimate the surface brightness of subcluster B to be $-0.344 \pm 0.028 \mathrm{MJy} \mathrm{sr}^{-1}$ at $140 \mathrm{GHz}$ and $0.052 \pm 0.029 \mathrm{MJy} \mathrm{sr}^{-1}$ at $268 \mathrm{GHz}$, where the errors represent only measurement uncertainties.

In addition to the best-fit model, we also compute the surface brightness toward sub-cluster B by directly integrating our deconvolved images, which are shown in Figure 4. As described in Section 4.1, the deconvolved images have no sensitivity to the DC signal level. As a result, we determine the DC signal level of the deconvolved images using the best-fit model. Specifically, we add a signal offset to the deconvolved images so that the average signal level within the $4^{\prime} \times 4^{\prime}$ region we use to constrain the model is equal to the average signal level of the best-fit model within the same region. We exclude the $1^{\prime}$ diameter aperture centered on sub-cluster $B$ in this calculation to avoid any potential bias in the surface brightness we derive within that aperture. This direct integration yields average surface brightnesses of $-0.341 \pm 0.027 \mathrm{MJy} \mathrm{sr}^{-1}$ and $0.095 \pm 0.049 \mathrm{MJy} \mathrm{sr}^{-1}$, respectively, where we have again estimated the uncertainties using the 1000 noise realizations. As with the model derived results, we used a KS test to determine if the distribution of 1000 values is consistent with Gaussian, and we find PTEs of 0.75 and 0.57 at 140 and $268 \mathrm{GHz}$, respectively. We note that these surface brightness values are consistent with those derived from the best-fit model, although there is significantly more measurement uncertainty on the $268 \mathrm{GHz}$ value. This additional uncertainty is a result of the significant large-angular-scale atmospheric noise in those data, which is amplified by the deconvolution of the signal transfer function.

\subsection{Systematic Uncertainties}

First, we note that our flux calibration is accurate to $5 \%$ at $140 \mathrm{GHz}$, and to $10 \%$ at $268 \mathrm{GHz}$ (Sayers et al. 2012). We have included these uncertainties in our systematic error budget.

To estimate the systematic errors due to the modeldependence of our results, we repeat our analysis of computing model-based and directly integrated surface brightnesses toward sub-cluster B at both 140 and $268 \mathrm{GHz}$ using a range of different models, with a summary of the results in Table 1 . First, we 
Table 1

Variations in Sub-cluster B's Surface Brightness Due to Possible Changes in Our Analysis Method

\begin{tabular}{|c|c|c|c|}
\hline \multicolumn{2}{|c|}{ Model-derived } & \multicolumn{2}{|c|}{ Direct Integration } \\
\hline $\begin{array}{l}140 \mathrm{GHz} \\
\left(\mathrm{MJy} \mathrm{sr}^{-1}\right)\end{array}$ & $\begin{array}{c}268 \mathrm{GHz} \\
\left(\mathrm{MJy} \mathrm{sr}^{-1}\right)\end{array}$ & $\begin{array}{c}140 \mathrm{GHz} \\
\left(\mathrm{MJy} \mathrm{sr}^{-1}\right)\end{array}$ & $\begin{array}{c}268 \mathrm{GHz} \\
\left(\mathrm{MJy} \mathrm{sr}^{-1}\right)\end{array}$ \\
\hline \multicolumn{4}{|c|}{ Nominal Values from Baseline Model } \\
\hline$-0.344 \pm 0.028$ & $0.052 \pm 0.029$ & $-0.341 \pm 0.027$ & $0.095 \pm 0.049$ \\
\hline \multicolumn{4}{|c|}{ Variations due to Model Choice } \\
\hline $\begin{array}{l}\text { Vary pseudo-Con } \\
\pm 0.012 \\
( \pm 0.4 \sigma)\end{array}$ & $\begin{array}{l}\text { ncertainties } \\
\quad \pm 0.009 \\
\quad( \pm 0.3 \sigma)\end{array}$ & $\begin{array}{l} \pm 0.006 \\
( \pm 0.2 \sigma)\end{array}$ & $\begin{array}{c} \pm 0.004 \\
( \pm 0.1 \sigma)\end{array}$ \\
\hline $\begin{array}{l}\text { Vary region used } \\
\leqslant 0.003 \\
(\leqslant 0.1 \sigma)\end{array}$ & $\begin{array}{c}\leqslant 0.019 \\
(\leqslant 0.7 \sigma)\end{array}$ & $\begin{array}{c}\leqslant 0.003 \\
(\leqslant 0.1 \sigma)\end{array}$ & $\begin{array}{c}\leqslant 0.024 \\
(\leqslant 0.5 \sigma)\end{array}$ \\
\hline $\begin{array}{l}\text { Model with no ps } \\
0.028 \\
(1.0 \sigma)\end{array}$ & $\begin{array}{c}\text { plates at B, C, an } \\
0.026 \\
(0.9 \sigma)\end{array}$ & $\begin{array}{c}0.016 \\
(0.6 \sigma)\end{array}$ & $\begin{array}{l}0.015 \\
(0.3 \sigma)\end{array}$ \\
\hline $\begin{array}{l}F \text {-test decision tr } \\
0.007 \\
(0.3 \sigma)\end{array}$ & $\begin{array}{c}\text { equal to } 0.04 \\
0.017 \\
(0.6 \sigma)\end{array}$ & $\begin{array}{l}0.001 \\
(0.0 \sigma)\end{array}$ & $\begin{array}{l}0.026 \\
(0.5 \sigma)\end{array}$ \\
\hline $\begin{array}{l}\text { Vary scale radius } \\
\leqslant 0.017 \\
(\leqslant 0.6 \sigma)\end{array}$ & $\begin{array}{r}-1.5 \\
\leqslant 0.026 \\
(\leqslant 0.9 \sigma)\end{array}$ & $\begin{array}{c}\leqslant 0.017 \\
(\leqslant 0.6 \sigma)\end{array}$ & $\begin{array}{c}\leqslant 0.022 \\
(\leqslant 0.4 \sigma)\end{array}$ \\
\hline \multicolumn{4}{|c|}{ Variations due to Aperture Choice } \\
\hline $\begin{array}{l}\text { Aperture centerec } \\
0.018 \\
(0.6 \sigma)\end{array}$ & $\begin{array}{c}\text { 012) coords } \\
0.019 \\
(0.7 \sigma)\end{array}$ & $\begin{array}{l}0.009 \\
(0.3 \sigma)\end{array}$ & $\begin{array}{c}0.003 \\
(0.1 \sigma)\end{array}$ \\
\hline $\begin{array}{l}\text { Aperture centerec } \\
0.019 \\
(0.7 \sigma)\end{array}$ & $\begin{array}{c}0.034 \\
(1.2 \sigma)\end{array}$ & $\begin{array}{c}0.010 \\
(0.4 \sigma)\end{array}$ & $\begin{array}{c}0.048 \\
(1.0 \sigma)\end{array}$ \\
\hline $\begin{array}{l}\text { Vary aperture dia } \\
\leqslant 0.029 \\
(\leqslant 1.0 \sigma)\end{array}$ & $\begin{array}{c}\leqslant 0.013 \\
(\leqslant 0.4 \sigma)\end{array}$ & $\begin{array}{c}\leqslant 0.024 \\
(\leqslant 0.9 \sigma)\end{array}$ & $\begin{array}{c}\leqslant 0.011 \\
(\leqslant 0.2 \sigma)\end{array}$ \\
\hline
\end{tabular}

Notes. Top block: best-fit surface brightnesses from the baseline model described in Section 5, and associated $1 \sigma$ uncertainties due to measurement noise only. Middle block: variations in the surface brightness of sub-cluster B based on our choice of model. We consider five different model fits to describe the SZ data. These models are explained in detail in the text, and we refer the reader there for more details. From left to right, the columns give the change in surface brightness at 140 and $268 \mathrm{GHz}$ for the model-derived and direct integration surface brightnesses. The top rows give these values in $\mathrm{MJy} \mathrm{sr}^{-1}$ and the bottom rows give these values relative to the measurement uncertainties in the top block. When noise variations to the models are considered, these values indicate the $1 \sigma$ range with a \pm symbol; when a range of model inputs are considered, these values show the magnitude of the maximum change with a $\leqslant$ symbol; and when a single alternative model is considered, these values show the magnitude of the change with no symbol. Based on these results, we add a systematic uncertainty equal to 1.0 times the measurement uncertainty for the model-derived values and equal to 0.6 times the measurement uncertainty for the direct integration values. Bottom block: variations in the surface brightness of sub-cluster B for different choices of aperture. From top to bottom the rows show the change relative to our nominal $1^{\prime}$ diameter aperture centered on the Ma et al. (2009) coordinates for (1) an aperture centered on the Limousin et al. (2012) coordinates, (2) an aperture centered on the $\mathrm{X}$-ray centroid, and (3) varying the aperture diameter between 0.67 and 1.5 for the aperture centered on the Ma et al. (2009) coordinates. All of these differences are consistent with the expected measurement noise fluctuations for the different aperture choices.

replace the baseline pseudo-Compton-y map we use in our model with a set of 20 realizations of the pseudo-Compton$y$ map that we generate according to the X-ray measurement uncertainties on the mean $T_{e}$ for each contbin region (see Section 4.2). Next, we constrain our baseline model using the $3^{\prime} \times 3^{\prime}$ and $5^{\prime} \times 5^{\prime}$ regions of the Bolocam images instead of the nominal $4^{\prime} \times 4^{\prime}$ region we use in Section 5. In addition, we consider an SZ model that does not include the pseudo-Compton- $y$ map, and instead only includes SZ templates centered on the sub-clusters. We repeat the $F$-test decision tree described in Section 5 to determine which of the sub-clusters require an SZ template for this model. We find that, without the pseudo-Compton- $y$ map, the data require $\mathrm{SZ}$ templates centered on sub-clusters $\mathrm{B}, \mathrm{C}$, and D. This fit produces a PTE of 0.64, indicating that the data are adequately described by this model. ${ }^{16}$ Furthermore, we reran the $F$-test decision tree with the PTE threshold increased by a factor of 2 to 0.04 . With this new threshold, the model consists of the pseudo-Compton- $y$ map with a single normalization, along with SZ templates centered on sub-clusters A and B

\footnotetext{
16 The adequacy of this somewhat simple and ad-hoc model in describing our data is likely due to Bolocam's coarse angular resolution, which largely blurs any sub-structures not well described by the smooth SZ templates. However, we note that this model requires twice as many free parameters as our baseline model in order to obtain an adequate fit according to our $F$-test decision tree.
} 
Table 2

SZ Surface Brightness

\begin{tabular}{|c|c|c|c|c|c|}
\hline $\begin{array}{l}\text { Frequency } \\
(\mathrm{GHz})\end{array}$ & $\begin{array}{c}\text { Best Fit } \\
\left(\mathrm{MJy} \mathrm{sr}^{-1}\right)\end{array}$ & $\begin{array}{l}\text { Measurement Err. } \\
\quad\left(\mathrm{MJy} \mathrm{sr}^{-1}\right)\end{array}$ & $\begin{array}{l}\text { Flux Err. } \\
\left(\mathrm{MJy} \mathrm{sr}^{-1}\right)\end{array}$ & $\begin{array}{l}\text { Modeling Err. } \\
\left(\mathrm{MJy} \mathrm{sr}^{-1}\right)\end{array}$ & $\begin{array}{l}\text { Total Err. } \\
\left(\mathrm{MJy} \mathrm{sr}^{-1}\right)\end{array}$ \\
\hline \multicolumn{6}{|c|}{ Sub-cluster B } \\
\hline \multicolumn{6}{|l|}{ Model Fits } \\
\hline 140 & -0.344 & 0.028 & 0.017 & 0.028 & 0.043 \\
\hline 268 & 0.052 & 0.029 & 0.005 & 0.029 & 0.041 \\
\hline \multicolumn{6}{|c|}{ Direct Integration } \\
\hline 140 & -0.341 & 0.027 & 0.017 & 0.016 & 0.036 \\
\hline 268 & 0.095 & 0.049 & 0.010 & 0.029 & 0.058 \\
\hline \multicolumn{6}{|c|}{ Sub-cluster C } \\
\hline \multicolumn{6}{|l|}{ Model Fits } \\
\hline 140 & -0.262 & 0.026 & 0.013 & 0.028 & 0.040 \\
\hline 268 & 0.217 & 0.039 & 0.022 & 0.029 & 0.053 \\
\hline \multicolumn{6}{|c|}{ Direct Integration } \\
\hline 140 & -0.270 & 0.026 & 0.014 & 0.016 & 0.034 \\
\hline 268 & 0.220 & 0.059 & 0.022 & 0.029 & 0.069 \\
\hline
\end{tabular}

Notes. The average surface brightness within a $1^{\prime}$ diameter aperture centered on sub-clusters B and C. From left to right the columns give the observing frequency, the best-fit average surface brightness, the measurement uncertainty on this value, the uncertainty on this value due to flux calibration, the uncertainty on this value due to the range of models we could have chosen to describe the data, and the total combined uncertainty, which is the quadrature sum of the previous three columns. For each sub-cluster, the top rows give the values we derive from the best-fit model of the SZ signal and the bottom rows give values we derive from direct integration of the deconvolved images.

(i.e., relative to the baseline model, an additional SZ template is required for sub-cluster A). Finally, we determine the effects of varying the scale radius of the profile used as a template of the SZ signal toward sub-cluster B. We vary the scale radius over a range of 0.67-1.5 times its nominal value, which corresponds to a scaling of the assumed mass of sub-cluster B by a factor of 0.3-3.4.

Considering this broad range of possible models that we could have chosen to describe our data, we find that the modelderived surface brightness of sub-cluster B never changes by more than 1.0 times the measurement uncertainties given in Section 6.1. For the surface brightness values obtained from direct integration of the deconvolved images, we find that the change is never larger than 0.6 times the uncertainties given in Section 6.1. As expected, the model-derived surface brightnesses have a stronger model dependence compared to the directly integrated surface brightnesses, although the latter still have a noticeable model-dependence due to the method by which we constrain the DC signal level of the deconvolved image. Based on these results, we conservatively include an additional systematic uncertainty of 1.0 times the measurement uncertainty for the model-derived surface brightnesses, and 0.6 times the measurement uncertainty for the directly integrated surface brightnesses. A full summary of our best-fit surface brightnesses, along with the full error budget on these values, is given in Table 2.

In addition to exploring how our choice of model affects our results, we also examine the effects of varying the aperture we use to compute the average surface brightness toward sub-cluster B. First, we examine three possible choices for the location of sub-cluster B: (1) the location given by Ma et al. (2009), based on the distribution of galaxies $(7: 17: 30.0,+37: 45: 35)$; (2) the location given by Limousin et al. (2012), based on the matter distribution $(7: 17: 30.2,+37: 45: 15)$; and (3) the location of the X-ray brightness centroid $(7: 17: 31.4,+37: 45: 29)$. Our nominal analysis uses the Ma et al. (2009) coordinates, and we give the changes in surface brightness when we use the other two possible apertures in Table 1. Compared to the measurement uncertainties given in Section 6.1, the surface brightnesses we measure in these new apertures differ by less than $\leqslant 1.2 \sigma$, with a median of $\simeq 0.7 \sigma$. The three sets of coordinates are separated from each other by $\simeq 20^{\prime \prime}$, which is a significant fraction of the aperture radius of $30^{\prime \prime}$, and means that less than $50 \%$ of the area enclosed by one aperture is also enclosed by another aperture. Consequently, completely uncorrelated measurement noise between any given pair of apertures will produce surface brightnesses that differ by $\simeq 1 \sigma$. Therefore, the differences in surface brightness that we measure between these aperture locations are consistent with the expectation due to noise fluctuations.

We also examine the effects of varying the diameter of the aperture from 0.67 to 1.5 (compared to the nominal diameter of $1^{\prime}$ ), and again find results that are consistent within $1 \sigma$. As with the different aperture locations, this is consistent with the variations that we expect due to uncorrelated measurement noise between the aperture choices, and indicates that variations in the location, or diameter, of the aperture we use to measure the SZ surface brightness result in differences consistent with measurement noise. We therefore conclude that our results are not sensitive to the exact choice of aperture, and we do not include any additional systematic error in our overall noise budget. We note that in all cases the apertures are comparable in size to the Bolocam PSF, and there is consequently some signal leakage from outside to inside the apertures and vice versa. Furthermore, the separation between the apertures centered on sub-clusters B and C is also comparable to the size of the Bolocam PSF, and so there is some signal leakage between apertures. Although we are not able to account for this signal leakage in our analysis, the consistency of our results using various aperture positions and diameters indicates that the leakage is below our measurement uncertainties. 


\subsection{Comparison to Previous Results}

We note that the $140 \mathrm{GHz}$ surface brightnesses we find for sub-cluster B are slightly different compared to the values reported in M12, although identical Bolocam data is used for both analyses. Our model-derived surface brightness of $-0.344 \pm 0.028 \mathrm{MJy} \mathrm{sr}^{-1}$ is more than $1 \sigma$ lower than the M12 value of $-0.293 \pm 0.030 \mathrm{MJy} \mathrm{sr}^{-1} .^{17}$ More than half of this difference is due to a minor error in the analysis presented in M12. The total flux densities given in Table 3 of M12 were mistakenly computed from the average surface brightness within a 1.5 aperture, rather than the $1^{\prime}$ aperture claimed in the text of M12 and also used in our present analysis. The remaining difference between our current surface brightness values and the ones presented in M12 is due to minor changes in our assumed model of the SZ signal. First, M12 constrained the normalization of the pseudo-Compton- $y$ map separately at 140 and $268 \mathrm{GHz}$, compared to the joint constraint we use in our present analysis. In addition, M12 assumed that the SZ template centered on sub-cluster B had a Gaussian profile, compared to the more physically motivated profile we use in this analysis, with a shape described by the best-fit profile to a sample of 45 clusters observed with Bolocam (Sayers et al. 2013a).

Furthermore, we note that, in our current analysis, the modelderived surface brightness agrees quite well with the surface brightness we obtain from a direct integration of the deconvolved image. This result is in contrast to the measurements presented in M12, where the two values differed by slightly more than $1 \sigma$. This change is due to differences in how the DC signal offset of the deconvolved images is computed. M12 computed the DC signal offset based on a fit of the average profile determined by Arnaud et al. (2010) to the full $140 \mathrm{GHz}$ Bolocam data set. Although the fit quality of this single profile is not particularly poor, with a PTE of 0.07 , the adequacy of using a single profile to describe a complex merging system like MACS $\mathrm{J} 0717.5+3745$ is questionable. Therefore, as described above, for this analysis we choose to constrain the DC signal offset of the $140 \mathrm{GHz}$ deconvolved image using our nominal model of the SZ signal (a pseudo-Compton- $y$ map with an additional SZ component centered on sub-cluster B). Not surprisingly, this change in our estimate of the DC signal level results in a better agreement between the model-derived and directly integrated surface brightnesses.

\section{MEASUREMENT OF THE SZ SPECTRUM TOWARD SUB-CLUSTER C}

M12 computed the SZ surface brightness toward both subcluster B and sub-cluster $\mathrm{C}$. The latter measurement was motivated primarily by the fact that $\mathrm{Ma}$ et al. (2009) identified sub-cluster $\mathrm{C}$ as the most massive component of MACS $\mathrm{J} 0717.5+3745$, along with the fact that sub-cluster $\mathrm{C}$ is coincident with the highest surface brightness in the $268 \mathrm{GHz}$ Bolocam image. Therefore, although our $F$-test decision tree indicates that our data do not require a component in addition to the thermal SZ template toward sub-cluster C, we again measure its SZ surface brightness. For these measurements we add an SZ template centered on sub-cluster $\mathrm{C}$ to our model, to ensure that the model has enough freedom to describe any possible deviations from a purely thermal SZ spectrum. We again estimate the SZ surface brightness using a $1^{\prime}$ diameter aperture

\footnotetext{
17 Table 3 of M12 lists a total flux density of $-19.5 \pm 2.0 \mathrm{mJy}$, which corresponds to a surface brightness of $-0.293 \pm 0.030 \mathrm{MJy} \mathrm{sr}^{-1}$.
}

centered on the coordinates from Ma et al. (2009), with the results given in Table 2. As with sub-cluster B, we estimate the uncertainties on these surface brightnesses using our 1000 noise realizations, and we again find that the distribution of values is consistent with Gaussian noise. In addition, we estimate the systematic uncertainty due to our choice of model using the same formalism described for sub-cluster B in Section 6.2. We find systematic errors consistent with those for sub-cluster B, and therefore adopt identical values for sub-cluster $\mathrm{C}$. Finally, we note that the $140 \mathrm{GHz}$ brightness values differ from those derived in M12 by roughly the same amounts as for sub-cluster $\mathrm{B}$, with the differences due to the same reasons described in detail in Section 6.3.

We do not attempt to constrain the SZ brightness toward either sub-cluster A or sub-cluster D. We do not consider sub-cluster A because it is not strongly detected in either Bolocam data set. We do not consider sub-cluster D because it is not separately resolved from sub-cluster C, due to Bolocam's coarse angular resolution, and therefore any estimate of sub-cluster D's SZ brightness would be highly correlated with our estimate of subcluster C's SZ brightness.

\section{PECULIAR VELOCITY CONSTRAINTS}

Using our two-band measurements of the SZ surface brightness toward sub-clusters $\mathrm{B}$ and $\mathrm{C}$, we are able to place constraints on the properties of the ICM of each sub-cluster. Based on the equations presented in Section 2, the total SZ brightness depends on four quantities related to the cluster ICM: $f\left(v, T_{e}\right), y, \tau_{e}$, and $v_{z}$. Our two-band SZ surface brightness measurements are insufficient to constrain all of these quantities, and so we therefore make the assumption that the ICM within each sub-cluster is isothermal and equal to the X-ray spectroscopic temperature determined within the same $1^{\prime}$ diameter apertures that we use to measure the SZ surface brightness. As a result, $f\left(v, T_{e}\right)$ is fully constrained by the Chandra-and-XMM-measured $T_{e}$, and from Equations (4) and (2) we have $y=\tau_{e} k_{B} T_{e} m_{e}^{-1} c^{-2}$, meaning that $y$ and $\tau_{e}$ are not independent. Therefore, we are left with two free parameters to constrain using the two-band Bolocam surface brightnesses, either $\tau_{e}$ and $v_{z}$ or $y$ and $v_{z}$ (in practice we constrain $Y_{\text {int }}$ and $v_{z}$, where $Y_{\text {int }}=y \Delta \Omega$, and $\Delta \Omega$ is equal to the solid angle of our $1^{\prime}$ aperture). In all of the fits, we compute the band-averaged values of $f\left(v, T_{e}\right)$ for a given $T_{e}$ using the full Bolocam bandpasses rather than a single effective band center.

Using our X-ray measured $T_{e}$, along with our SZ surface brightnesses, we then perform a grid search to constrain the values of $v_{z}$ and $Y_{\text {int }}$ for sub-clusters $\mathrm{B}$ and $\mathrm{C}$. For these constraints, we use the best-fit SZ surface brightness values from Table 2, along with the total uncertainties in the far-right column of that table. Therefore, we fully include not only measurement uncertainties, but also flux calibration uncertainties, and possible systematic uncertainties due to our choice of model to describe the SZ signal. Because the noise in our SZ surface brightness measurements is indistinguishable from Gaussian, we compute likelihoods based on a Gaussian distribution. When fitting the SZ spectra, we marginalize over the range of $T_{e}$ values allowed by the $\mathrm{X}$-ray data, relying on the $\mathrm{C}$ statistic to give a likelihood for each temperature in the range $2-40 \mathrm{keV}$. A summary of our results for both sub-clusters is given in Table 3 and Figures 5 and 6 , and we highlight some of these results below.

For sub-cluster B we find a best-fit $v_{z}=+3450 \mathrm{~km} \mathrm{~s}^{-1}$ using the SZ surface brightnesses we determine from the model fit to our data and a best-fit $v_{z}=+2550 \mathrm{~km} \mathrm{~s}^{-1}$ using the SZ surface brightnesses we determine from direct integration of our 

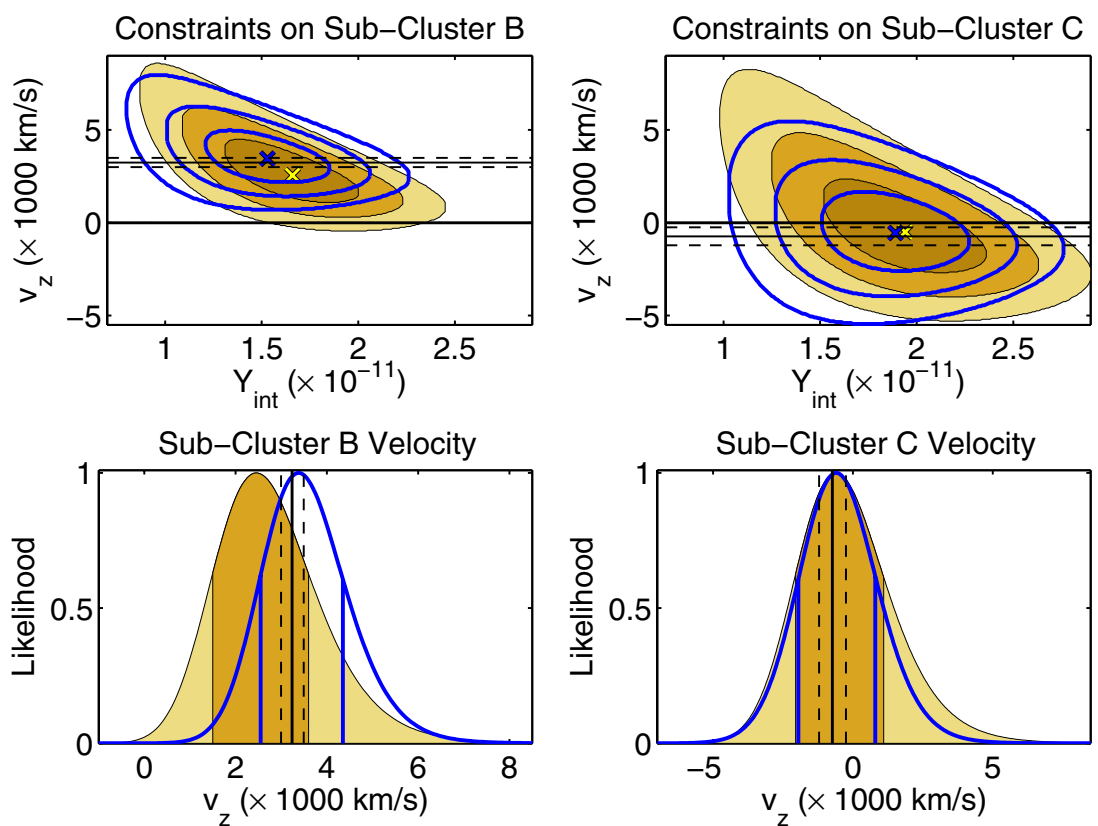

Figure 5. Our SZ-derived constraints on the ICM toward sub-cluster B (left) and sub-cluster C (right). The top row shows two-dimensional confidence regions for the values of $Y_{\text {int }}$ and $v_{z}$, with contours drawn at $1 \sigma, 2 \sigma$, and $3 \sigma$ for a two-parameter likelihood (e.g., $1 \sigma$ corresponds to $\Delta \chi^{2}=2.30$ ). The bottom row shows marginalized one-dimensional likelihoods for $v_{z}$, with vertical lines drawn at $\pm 1 \sigma$ (corresponding to $\Delta \chi^{2}=1$ ). In all cases blue corresponds to the constraints from the model-derived SZ surface brightnesses, and yellow corresponds to the constraints from the SZ surface brightnesses we derive from direct integration of the deconvolved images. The solid black line represents the best-fit velocity derived by Ma et al. (2009) based on optical spectroscopy, and the dashed lines show the corresponding $1 \sigma$ confidence region around their best-fit.

(A color version of this figure is available in the online journal.)
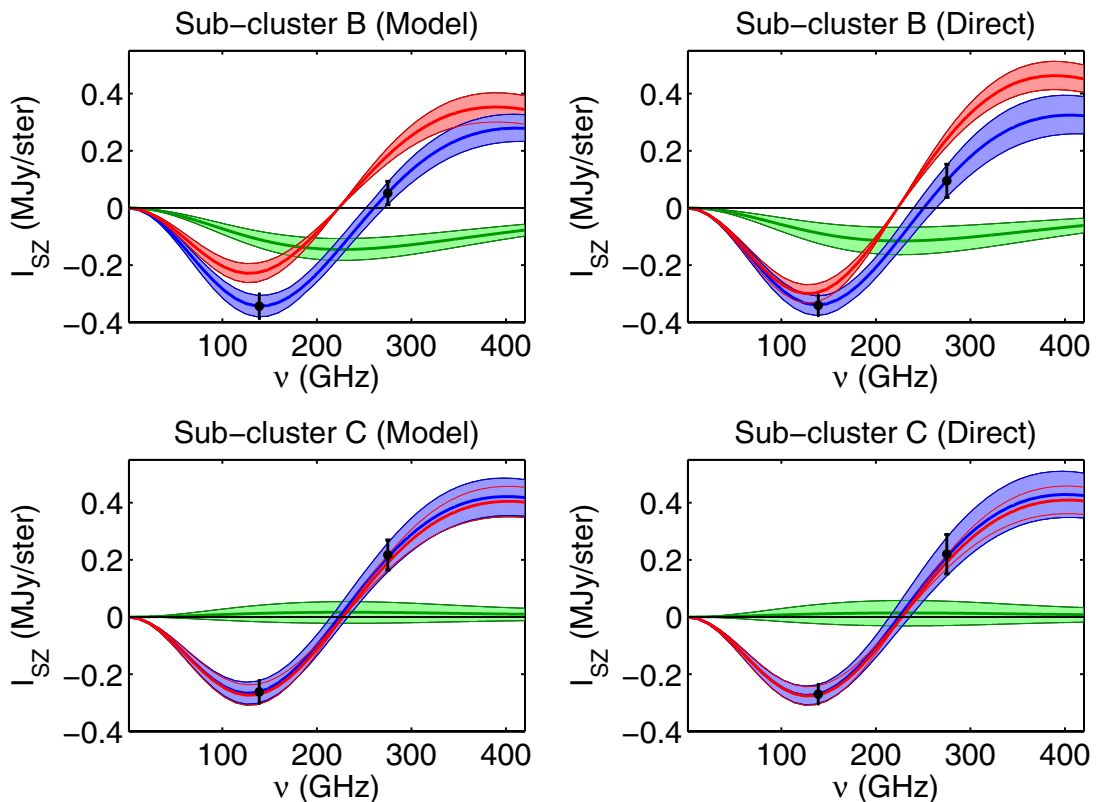

Figure 6. Our best-fit SZ spectra. The top row shows the fits to sub-cluster B, and the bottom row shows the fits to sub-cluster C. The left column shows the SZ surface brightnesses we determine from the model fit, and the right column shows the SZ surface brightnesses we determine via direct integration of the deconvolved images. The best-fit thermal-SZ-only spectrum is shown in red, the best-fit kinetic SZ spectrum is shown in green, and the best-fit thermal plus kinetic SZ spectrum is shown in blue, with the widths showing the $1 \sigma$ confidence region of the fits. We include relativistic corrections in all of the spectra.

(A color version of this figure is available in the online journal.)

deconvolved images. Both of these values are consistent with the value of $+3238 \mathrm{~km} \mathrm{~s}^{-1}$ determined by Ma et al. (2009), based on optical spectroscopy under the assumption that the peculiar velocity of the entire cluster is 0 along the line-of-sight (see Figure 5). The $1 \sigma$ uncertainties about these best-fit velocities are similar for both the model-derived and direct integration results, and are $\lesssim 1000 \mathrm{~km} \mathrm{~s}^{-1}$. We also compute the probability of $v_{z} \geqslant 0$, and obtain values of $\left(1-\operatorname{Prob}\left[v_{z} \geqslant 0\right]\right)=1.3 \times 10^{-5}$ and $2.2 \times 10^{-3}$ for the model-derived and direct integration SZ surface brightnesses, respectively (see the bottom panels of Figure 5). For a Gaussian distribution, these one-sided probabilities correspond to a difference from $v_{z}=0$ of $4.2 \sigma$ and $2.9 \sigma$, respectively. For sub-cluster $\mathrm{C}$ we find a best-fit $v_{z}$ of $\simeq-500 \mathrm{~km} \mathrm{~s}^{-1}$ from both the model fit and direct integration 
Table 3

Peculiar Velocity Constraints

\begin{tabular}{|c|c|c|c|c|}
\hline & $\begin{array}{c}T_{e} \\
(\mathrm{keV})\end{array}$ & $\begin{array}{c}\text { Optical } \\
\left(\mathrm{km} \mathrm{s}^{-1}\right)\end{array}$ & $\begin{array}{l}\text { SZ Model Fit } \\
\quad\left(\mathrm{km} \mathrm{s}^{-1}\right)\end{array}$ & $\begin{array}{l}\text { SZ Direct Integration } \\
\left(\mathrm{km} \mathrm{s}^{-1}\right)\end{array}$ \\
\hline Sub-cluster B & $11.4_{-0.5}^{+0.5}$ & $+3238_{-242}^{+252}$ & $+3450_{-900}^{+900}\left(1-\operatorname{Prob}\left[v_{z} \geqslant 0\right]=1.3 \times 10^{-5}\right)$ & $+2550_{-1050}^{+1050}\left(1-\operatorname{Prob}\left[v_{z} \geqslant 0\right]=2.2 \times 10^{-3}\right)$ \\
\hline Sub-cluster C & $19.9_{-1.4}^{+1.5}$ & $-733_{-478}^{+486}$ & $-550_{-1400}^{+1350}\left(1-\operatorname{Prob}\left[v_{z} \leqslant 0\right]=3.7 \times 10^{-1}\right)$ & $-500_{-1550}^{+1600}\left(1-\operatorname{Prob}\left[v_{z} \leqslant 0\right]=4.3 \times 10^{-1}\right)$ \\
\hline
\end{tabular}

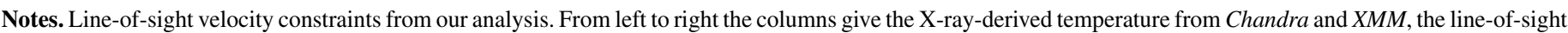

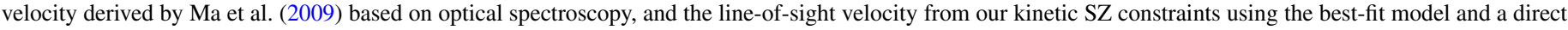

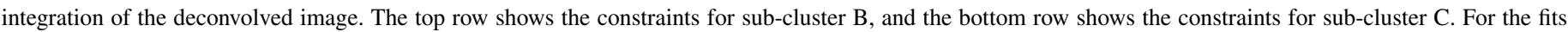

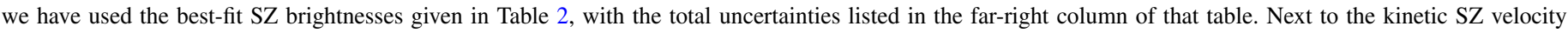

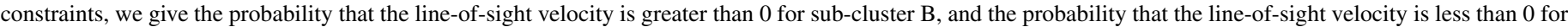
sub-cluster C.

of the deconvolved image, which is fully consistent with both the value of $-733 \mathrm{~km} \mathrm{~s}^{-1}$ determined by Ma et al. (2009) and with zero velocity. We note that the uncertainties on the value of $v_{z}$ for sub-cluster $\mathrm{C}$ are $\simeq 50 \%$ larger compared to sub-cluster $\mathrm{B}$. This increase is due entirely to the higher temperature of sub-cluster $\mathrm{C}$. This higher temperature produces a smaller value of $\tau_{e}$ for a fixed value of $Y_{\text {int }}$ and therefore a correspondingly lower kinetic SZ signal for a fixed value of $v_{z}$.

We note that the difference between our best fit $v_{z}$ and the best fit $v_{z}$ from Ma et al. (2009) is quite small for both subclusters $(0.23 \sigma$ and $0.13 \sigma$ for the model-derived results for subclusters $\mathrm{B}$ and $\mathrm{C}$, and $0.64 \sigma$ and $0.14 \sigma$ for the direct-integration results for sub-clusters $\mathrm{B}$ and $\mathrm{C}$ ). The random probability of obtaining such results from two independent measurements of two independent parameters is $2 \%$ for our model-derived results and $6 \%$ for our direct-integration results. These probabilities are small, but they are not small enough to cause significant concern. In addition, our intentionally conservative estimates of the uncertainties due to our choice of SZ model have likely resulted in over-estimated errors on the SZ brightness, thus rendering the good agreement between our results and those of Ma et al. (2009) more likely.

\section{DISCUSSION}

\subsection{Differences Compared to the Results in M12}

Compared to the results presented in M12, our best-fit values of $v_{z}$ for sub-cluster B are somewhat lower $\left(+3450 \mathrm{~km} \mathrm{~s}^{-1}\right.$ and $+2550 \mathrm{~km} \mathrm{~s}^{-1}$, compared to $+4640 \mathrm{~km} \mathrm{~s}^{-1}$ and $+3600 \mathrm{~km} \mathrm{~s}^{-1}$ for the model-derived and direct integration surface brightnesses, respectively). This is mainly due to an increase in the best-fit surface brightness at $268 \mathrm{GHz}$ as a result of the additional data we use in our present analysis. In contrast, our best-fit values of $v_{z}$ for sub-cluster $\mathrm{C}$ are smaller in magnitude compared to M12 $\left(-550 \mathrm{~km} \mathrm{~s}^{-1}\right.$ and $-500 \mathrm{~km} \mathrm{~s}^{-1}$ versus $-3720 \mathrm{~km} \mathrm{~s}^{-1}$ and $-4120 \mathrm{~km} \mathrm{~s}^{-1}$ for the model-derived and direct integration surface brightnesses, respectively). These differences are again driven by the additional $268 \mathrm{GHz}$ data we use in our current analysis, which indicates that sub-cluster $\mathrm{C}$ is dimmer compared to the analysis of M12. However, we emphasize that all of our measured values of $v_{z}$ are consistent to within $1 \sigma$ of the values presented in M12, and there is no tension between the two results.

Our uncertainties on the value of $v_{z}$ for sub-cluster B are a factor of $\simeq 3$ smaller than the uncertainties reported in M12. This improvement is almost entirely due to the significant amount of additional $268 \mathrm{GHz}$ data we use in this analysis and the corresponding factor of $\simeq 2.5$ decrease in the uncertainties on the $268 \mathrm{GHz}$ surface brightness. A small additional improvement is driven by the lower value of $T_{e}$ found in our present analysis, which for a fixed $Y_{\text {int }}$ corresponds to a larger $\tau_{e}$ and therefore a larger kinetic SZ signal for a fixed $v_{z}$. In contrast, our uncertainties on $v_{z}$ for sub-cluster $\mathrm{C}$ have only decreased by a factor of $\simeq 2$ compared to M12, even though there is a similar reduction in the $268 \mathrm{GHz}$ measurement uncertainties. This difference relative to sub-cluster B is driven by our best-fit value of $v_{z}$, which is significantly larger (less negative) than the results in M12. As a result, the best-fit value of $Y_{\text {int }}$ is smaller, and therefore the best-fit value of $\tau_{e}$ is smaller. Consequently, for a given change in $v_{z}$, the corresponding change in the kinetic SZ surface brightness is also smaller, resulting in less constraining power on the value of $v_{z}$.

In contrast to the analysis presented in M12, note that we include additional systematic uncertainties in our derived SZ surface brightnesses due to differences based on the range of models we could have chosen to describe the SZ signal. These systematic uncertainties increase the total error estimate on the model-derived and directly integrated SZ surface brightnesses by $\simeq 40 \%$ and $\simeq 20 \%$, respectively. We perform fits of $v_{z}$ without including this additional systematic error, and verify that the fractional improvement in our constraints matches these values. Therefore, the model-dependence of our SZ data results in a non-negligible degradation of our constraining power on $v_{z}$.

\subsection{Limitations to Our Kinetic SZ Constraints}

Given the range of multi-wavelength data that we use to place constraints on $v_{z}$, we also estimate how each of these data sets contribute to our overall uncertainties. First, as noted in Section 4.3, previous results have indicated that there is a systematic difference in the temperatures derived from Chandra and $X M M$, and the temperatures we measure are in general agreement with this systematic difference. At this point, the cause of this difference has not been conclusively demonstrated. Due to the lack of a conclusive understanding of the difference, combined with the fact that the difference between the X-ray temperatures we derive from the two observatories is of modest statistical significance, we choose to constrain the electron temperatures via the joint likelihood from the Chandra and $X M M$ data. If we adopt the $X M M$-only values of $T_{e}$, then we find best-fit values of $v_{z}$ equal to $+3300 \mathrm{~km} \mathrm{~s}^{-1}$ and $+2450 \mathrm{~km} \mathrm{~s}^{-1}$ for the model fit and direct integration of subcluster $\mathrm{B}$ and $-450 \mathrm{~km} \mathrm{~s}^{-1}$ and $-400 \mathrm{~km} \mathrm{~s}^{-1}$ for the model fit and direct integration of sub-cluster C. If we adopt the Chandra-only values of $T_{e}$, then we find best-fit values $v_{z}$ equal to $+4000 \mathrm{~km} \mathrm{~s}^{-1}$ and $+2900 \mathrm{~km} \mathrm{~s}^{-1}$ for the model fit and direct integration of sub-cluster B and $-550 \mathrm{~km} \mathrm{~s}^{-1}$ and $-450 \mathrm{~km} \mathrm{~s}^{-1}$ for the model fit and direct integration of sub-cluster C. For 
sub-cluster B, the Chandra-only temperatures yield line-ofsight velocities that differ by $\simeq 0.5 \sigma$, but all of the other values are statistically indistinguishable from our results in Table 3. Therefore, we conclude that X-ray calibration uncertainties do not strongly affect our constraints on $v_{z}$. We further note that the significance of our kinetic SZ measurement from $v_{z}=0$ is nearly independent of the exact value of $T_{e}$ and the slight differences in $v_{z}$ for the different temperatures are due to the inverse relationship between $T_{e}$ and $\tau_{e}$ for a fixed $y$, coupled with the inverse relationship between $v_{z}$ and $\tau_{e}$ for a fixed kinetic SZ surface brightness.

To assess the impact of the X-ray uncertainties on $T_{e}$, we also rerun all of our fits with vanishing uncertainties on the X-ray derived $T_{e}$. Even in the case of sub-cluster $\mathrm{C}$, when using the Chandra-only measurement with uncertainties of $T_{e}+7.8 \mathrm{keV}$, the derived uncertainties on $v_{z}$ increase by only $\simeq 10 \%$ when using the measured uncertainties instead of assuming that the uncertainty on $T_{e}$ is equal to 0 . Therefore, the $\mathrm{X}$-ray uncertainties are not significant in our overall error budget on $v_{z}$.

To determine the effect of the CIB on our measurement of $v_{z}$, we also compute the SZ brightness under the assumption that the CIB is completely and noiselessly subtracted from the data. Specifically, compared to our default noise realizations, we remove the noise from the undetected CIB, along with our uncertainties on the subtracted CIB (see the Appendix). This results in a negligible change in the $140 \mathrm{GHz}$ surface brightness uncertainties, and $\mathrm{a} \simeq 10 \%-20 \%$ reduction in the $268 \mathrm{GHz}$ surface brightness uncertainties. There is a corresponding $\simeq 10 \%-20 \%$ reduction in our derived uncertainties on $v_{z}$. In addition, we estimate the potential bias that would result from not subtracting any of the Bolocam or SPIRE-detected galaxies from our $268 \mathrm{GHz}$ data. We find that our best-fit $268 \mathrm{GHz}$ surface brightness values change by $\simeq 10 \%$, indicating that the bright sources in the CIB produce a non-negligible bias in the SZ surface brightnesses we measure. Therefore, at our sensitivities, the CIB has a noticeable effect on our kinetic SZ measurement, and is more significant than uncertainties on the X-ray-derived electron temperature.

We also perform fits under the assumption that primary CMB fluctuations are perfectly subtracted from our data by removing them from our noise realizations. This does not produce a noticeable change in the $268 \mathrm{GHz}$ surface brightness constraints, but does improve the $140 \mathrm{GHz}$ surface brightness constraints by $\simeq 5 \%$, with a corresponding improvement in our derived constraints on $v_{z}$. Therefore, noise from primary CMB fluctuations has an effect on our kinetic SZ measurements that is smaller than, but comparable to, noise from CIB fluctuations. This mild sensitivity to primary CMB fluctuations is due to the relative shallowness of our $140 \mathrm{GHz}$ data, which have an rms of $\simeq 30 \mu \mathrm{K}_{\mathrm{CMB}}$ arcmin (see Table 1 of Sayers et al. 2013a).

Examining the error budget in Table 2, the dominant uncertainties are associated with SZ measurement noise and the exact choice of model used to describe the SZ data, although we note that uncertainties due to absolute flux calibration are only a factor of $\simeq 2$ smaller. As detailed in Section 4.1, a model is required to interpret our SZ data because the large-angular scale atmospheric noise necessitates a high-pass filtering of the data, which removes the signal on large angular scales. As a result, a spatial model of the SZ is the only way to recover this large-scale signal in order to obtain an absolute surface brightness. Therefore, this modeling uncertainty is a direct result of the measurement noise in the SZ data, and is not a fundamental limitation. Deeper SZ data would provide a significant improvement to our kinetic SZ measurement, although these deeper SZ data will require better absolute flux calibration, are likely to require an improved subtraction of the CIB (and possibly the primary CMB fluctuations), and may require an improved understanding the X-ray temperature calibration or the line-of-sight temperature structure (e.g., Chluba et al. 2012; Prokhorov \& Colafrancesco 2012).

\subsection{Additional Potential Sources of Bias}

Our analysis constrains the line-of-sight peculiar velocities of two of the sub-clusters of MACS J0717.5+3745 via a measurement of the SZ surface brightnesses within small apertures centered on these sub-clusters. Due to the complex dynamics in MACS J0717.5+3745, the SZ signal within these apertures may not be sourced by gas bound to a single sub-cluster with a single coherent bulk velocity. However, as described in Section 3, the X-ray data show that sub-cluster B does appear to have a relatively intact core region. Therefore, at least for sub-cluster B, the assumption of a single bound ICM appears to be justified. Sub-cluster $\mathrm{C}$ seems to be more disturbed, and this assumption may not be valid for that region.

In addition to possible merger-induced gas inhomogeneities, there are also likely to be line-of-sight projection effects that cause the SZ signal within a single aperture to be sourced by the ICMs of multiple sub-clusters. In part to answer this question, Ruan et al. (2013) studied the SZ signal from a simulated triple-merger system in detail. Their simulated cluster is similar to MACS J0717.5+3745, and contains one sub-cluster with a velocity of $2500 \mathrm{~km} \mathrm{~s}^{-1}$. They used kinetic SZ measurements at 90 and $268 \mathrm{GHz}$ to constrain the line-of-sight velocities of the sub-clusters within the merger and found best-fit velocities that are consistent with the true velocities of the sub-clusters to within $\simeq 10 \%$. This is partly due to the fact that the SZ signal from the core of the sub-cluster of interest is significantly brighter than the SZ signal away from the core of other subclusters in projection. However, the merging sub-cluster also induces a small kinetic SZ signal in the ICMs of the other subclusters in projection with it, and this induced signal serves to bring the SZ-measured velocity into better agreement with the true velocity. These results indicate that at our current measurement precision, the bias in our measured velocities due to interactions and projection effects from other sub-clusters is likely to be statistically insignificant.

\subsection{Cosmological Implications}

Our kinetic SZ measurements are in good agreement with the spectroscopic measurements of Ma et al. (2009) and indicate that sub-cluster $\mathrm{B}$ is moving with a line-of-sight velocity of $\simeq 3000 \mathrm{~km} \mathrm{~s}^{-1}$ compared to the center of mass of the system. Ma et al. (2009) note that this value is close to the maximum expected velocity due to infall from infinity. For example, if sub-cluster B starts from rest at infinity, and if the main cluster has a mass of $1.5 \times 10^{15} M_{\odot}$, then sub-cluster B would need to be within $\simeq 1.5 \mathrm{Mpc}$ of the main cluster to reach an infall velocity of $3000 \mathrm{~km} \mathrm{~s}^{-1}$. This is in fairly good agreement with N-body simulations, which indicate that a relative velocity of $\simeq 3000 \mathrm{~km} \mathrm{~s}^{-1}$ is possible for a MACS J0717.5+3745-like cluster within the framework of the standard cosmological model. For example, Lee \& Komatsu (2010) showed that mergers with main cluster masses above $1 \times 10^{15} M_{\odot}$ at $z=0.5$ have a non-negligible probability of producing velocities larger than $3000 \mathrm{~km} \mathrm{~s}^{-1}$ when the sub-cluster is within the virial radius of the main cluster. In addition, the cluster studied by Ruan et al. (2013; see Section 9.3) was selected from a cosmological 
simulation of a $400 \mathrm{~h}^{-1} \mathrm{Mpc}$ cube, and one of its sub-clusters has a line-of-sight velocity of $2500 \mathrm{~km} \mathrm{~s}^{-1}$. Therefore, we conclude that while the velocity of sub-cluster B is large, it is not in any tension with the standard cosmological models.

\section{SUMMARY}

We detect an extended SZ signal toward MACS J0717.5+3745 at high significance in two observing bands with Bolocam (140 and $268 \mathrm{GHz}$ ). The $268 \mathrm{GHz}$ data also contain significant emission from dusty star forming galaxies. We subtract all of the galaxies brighter than $\simeq 1 \mathrm{mJy}$ using a combination of Herschel-SPIRE and Bolocam data, although both this subtraction, and the un-subtracted population of dimmer galaxies, produce a non-negligible amount of noise in our measurement of the SZ signal (see Section 9.2). Using a rigorous decision tree based on the application of the $F$-test, we find that a physically-motivated model composed of a Chandra-derived pseudo-Compton- $y$ map to describe the thermal SZ signal, plus an additional template centered on sub-cluster B with different normalizations at 140 and $268 \mathrm{GHz}$, is the minimum model that is adequate to describe our data. We note that sub-cluster B has a measured spectroscopic line-of-sight velocity of $+3200 \mathrm{~km} \mathrm{~s}^{-1}$ (Ma et al. 2009).

From this best-fit model, we compute the two-band SZ surface brightness toward sub-cluster $\mathrm{B}$, along with the most massive sub-cluster, C. We also compute the SZ surface brightness by directly integrating the Bolocam images, although the best-fit model is required to constrain the DC signal level of these images, which is filtered away by our data processing. For both the model-derived and directly integrated SZ surface brightnesses, we include uncertainties due to measurement noise and absolute flux calibration. In addition, we include an uncertainty due to the variations in derived surface brightnesses for a range of physically motivated models that we could have chosen to describe our data, and we find that this uncertainty is similar to our measurement uncertainty.

Using our measured SZ surface brightnesses toward subclusters $\mathrm{B}$ and $\mathrm{C}$, along with our $\mathrm{X}$-ray-derived electron temperatures for each sub-cluster, we constrain a spectral model consisting of thermal and kinetic SZ components (see Figure 6). For these fits, we assume that the ICM is isothermal within small apertures centered on each sub-cluster, and we include corrections for relativistic effects. We find that a thermal SZ signal is adequate to describe the SZ surface brightnesses of sub-cluster $C$, but that an additional kinetic SZ signal is required for sub-cluster B. From our model-derived SZ surface brightnesses, this kinetic SZ signal implies a line-ofsight velocity of $v_{z}=+3450 \mathrm{~km} \mathrm{~s}^{-1}$, whereas the directly integrated SZ surface brightnesses imply a line-of-sight velocity of $v_{z}=+2550 \mathrm{~km} \mathrm{~s}^{-1}$, both of which are in good agreement with the spectroscopic measurement of Ma et al. (2009; see Figure 5). From the model fit we find that $\left(1-\operatorname{Prob}\left[v_{z} \geqslant 0\right]\right)$ is $1.3 \times 10^{-5}$, which corresponds to being $4.2 \sigma$ from 0 for a Gaussian distribution. Similarly, from the direct integration of the SZ surface brightness, we find that $\left(1-\operatorname{Prob}\left[v_{z} \geqslant 0\right]\right)$ is $2.2 \times 10^{-3}$, which corresponds to being $2.9 \sigma$ from 0 for a Gaussian distribution.

We consider potential biases in our derived values of $v_{z}$ due to possible systematics in the X-ray derived $T_{e}$, and due to merger and projection effects as a result of the complex dynamics of this cluster, and we find that neither bias is likely to be significant compared to our measurement uncertainties. We find that raw SZ measurement sensitivity limits our constraints on $v_{z}$, and uncertainties from the X-ray data, the CIB, the $\mathrm{CMB}$, and flux calibration are sub-dominant, although deeper SZ measurements will likely be limited by some combination of these factors. Our data, combined with the results from $\mathrm{Ma}$ et al. (2009), indicate that sub-cluster B is moving with a lineof-sight velocity of $\simeq+3000 \mathrm{~km} \mathrm{~s}^{-1}$, a value that is high, but not in tension with standard cosmological models.

We acknowledge the assistance of: the day crew and Hilo staff of the Caltech Submillimeter Observatory, who provided invaluable assistance during data-taking for this data set; Kathy Deniston, Barbara Wertz, and Diana Bisel, who provided effective administrative support at Caltech and in Hilo; the Bolocam observations were partially supported by the Gordon and Betty Moore Foundation. J.S. was supported by NSF/AST-0838261, NASA/NNX11AB07G, and the Norris Foundation CCAT Postdoctoral Fellowship; support for T.M. was provided by NASA through Einstein Fellowship Program grant No. PF0-110077 awarded by the Chandra X-ray Center, which is operated by the Smithsonian Astrophysical Observatory for NASA under contract NAS8-03060; P.M.K. was supported by a NASA Postdoctoral Program Fellowship; N.C. was partially supported by a NASA Graduate Student Research Fellowship; A.M. was partially supported by NSF/AST-0838187 and NSF/AST-1140019; E.P. and J.A.S. were partially supported by NASA/NNX07AH59G; S.S. was supported by NASA Earth and Space Science Fellowship NASA/NNX12AL62H; K.U. acknowledges partial support from the National Science Council of Taiwan grant NSC100-2112-M-001-008-MY3 and from the Academia Sinica Career Development Award. A portion of this research was carried out at the Jet Propulsion Laboratory, California Institute of Technology, under a contract with the National Aeronautics and Space Administration. This research made use of the Caltech Submillimeter Observatory, which was operated at the time by the California Institute of Technology under cooperative agreement with the National Science Foundation (NSF/AST-0838261). This work is also based in part on observations made with Herschel, a European Space Agency Cornerstone Mission with a significant participation by NASA. Partial support for this work was provided by NASA through an award issued by JPL/Caltech.

Facilities: CSO, CXO, Herschel, XMM

\section{APPENDIX}

There is a significant amount of signal from unresolved dusty star-forming galaxies (e.g., Blain et al. 2002) in our $268 \mathrm{GHz}$ Bolocam map, and we describe our treatment of these galaxies in this Appendix. Unlike the resolved SZ signal we seek to measure, all of the dusty star-forming galaxies in our Bolocam image are unresolved. Therefore, to maximize our sensitivity to these unresolved sources, we process the $268 \mathrm{GHz}$ data using an adaptive principal component analysis (PCA) algorithm in place of the common-mode subtraction we use for our SZ analysis. For brevity, we refer to the maps generated by these reductions as the adaptive-PCA map and the common-mode-subtraction map. The details of the adaptivePCA algorithm we use for this analysis are given in $\mathrm{Wu}$ et al. (2012). This processing results in an adaptive-PCA map with a noise rms of $0.7 \mathrm{mJy}_{\text {beam }}{ }^{-1}$, which is approximately equal to the confusion noise from unresolved star-forming galaxies. We then subtract a template of the extended SZ signal from the adaptive-PCA map by fitting a generalized 

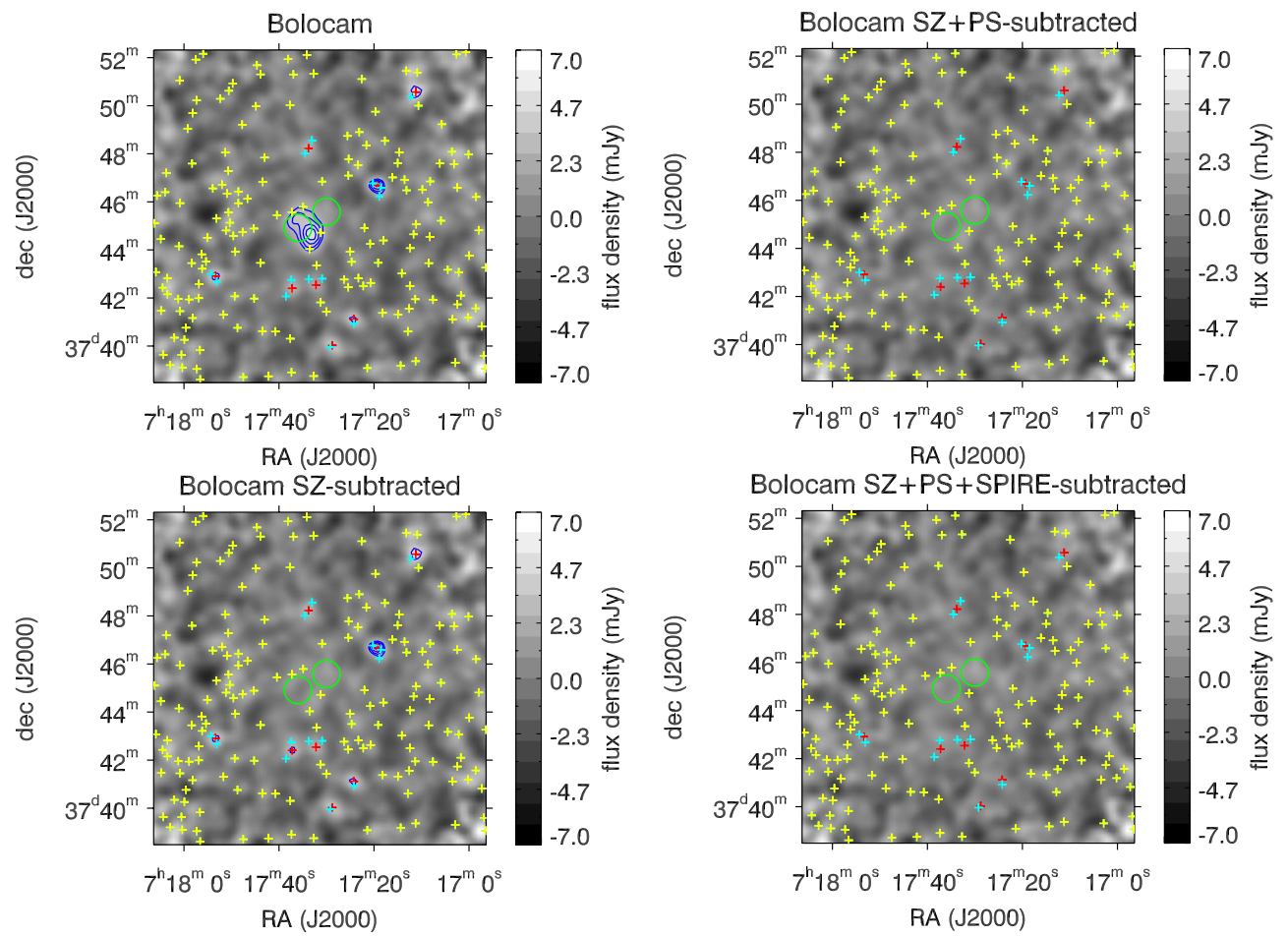

Figure 7. Bolocam $268 \mathrm{GHz}$ images we obtain from the adaptive-PCA reduction. Clockwise from upper left: nominal image, image after subtracting the best-fit extended-SZ template, image after also subtracting the eight unresolved sources detected by Bolocam, and image after also subtracting the 162 sources detected by SPIRE and extrapolated to Bolocam's band using a graybody fit. The blue contours show S/N, starting at 4 and separated by 1 . There are no contours around the bright regions in the corners of the images, which is due to the higher noise in these regions as a result of reduced integration time relative to the central region. Note that even in this heavily filtered image, an extended SZ signal is detected at high significance. The crosses show: Bolocam detections (red), likely SPIRE counterparts to those detections (cyan), and all other SPIRE detections with an extrapolated S/N $>2$ (yellow). The green circles show the $1^{\prime}$ diameter apertures centered on sub-cluster $\mathrm{C}$ (lower left) and sub-cluster B (upper right).

(A color version of this figure is available in the online journal.)

Table 4

Unresolved Sources Detected by Bolocam at $268 \mathrm{GHz}$

\begin{tabular}{|c|c|c|c|c|c|}
\hline $\begin{array}{l}\text { R.A. } \\
(\text { J2000) }\end{array}$ & $\begin{array}{l}\text { Decl. } \\
(\mathrm{J} 2000)\end{array}$ & $\begin{array}{l}\text { Flux Density } \\
\text { (mJy) }\end{array}$ & $\begin{array}{l}\text { De-boosted } \\
\text { (mJy) }\end{array}$ & $\begin{array}{l}\text { SPIRE Extrapolated } \\
(\mathrm{mJy})\end{array}$ & $\begin{array}{l}\text { SPIRE Distance } \\
\text { (") }\end{array}$ \\
\hline \multirow[t]{3}{*}{$7: 17: 19.4$} & $37: 46: 41$ & $7.6 \pm 0.9$ & $6.9 \pm 0.8$ & $6.2 \pm 0.5$ & 11 \\
\hline & & & & $1.6 \pm 0.2$ & 11 \\
\hline & & & & $0.4 \pm 0.1$ & 28 \\
\hline \multirow[t]{2}{*}{$7: 17: 53.4$} & $37: 42: 55$ & $5.2 \pm 1.2$ & $3.7 \pm 1.4$ & $2.2 \pm 0.1$ & 12 \\
\hline & & & & $1.4 \pm 0.1$ & 15 \\
\hline $7: 17: 24.3$ & $37: 41: 07$ & $5.3 \pm 1.1$ & $4.0 \pm 1.2$ & $3.7 \pm 0.6$ & 11 \\
\hline \multirow[t]{2}{*}{$7: 17: 37.3$} & $37: 42: 24$ & $3.9 \pm 0.9$ & $3.2 \pm 1.0$ & $1.2 \pm 0.1$ & 21 \\
\hline & & & & $2.5 \pm 0.5$ & 25 \\
\hline \multirow[t]{2}{*}{$7: 17: 32.2$} & $37: 42: 32$ & $3.6 \pm 0.9$ & $3.0 \pm 1.0$ & $0.5 \pm 0.1$ & 22 \\
\hline & & & & $0.8 \pm 0.1$ & 23 \\
\hline $7: 17: 11.2$ & $37: 50: 34$ & $6.3 \pm 1.4$ & $4.0 \pm 1.7$ & $3.0 \pm 0.1$ & 17 \\
\hline $7: 17: 28.8$ & $37: 40: 02$ & $5.3 \pm 1.3$ & $3.5 \pm 1.6$ & $5.3 \pm 0.3$ & 7 \\
\hline \multirow[t]{2}{*}{$7: 17: 33.8$} & $37: 48: 13$ & $3.5 \pm 0.9$ & $2.9 \pm 1.0$ & $2.9 \pm 0.5$ & 16 \\
\hline & & & & $0.6 \pm 0.1$ & 22 \\
\hline
\end{tabular}

Notes. Unresolved sources that we detect with an $\mathrm{S} / \mathrm{N} \geqslant 4$ in the Bolocam $268 \mathrm{GHz}$ data. From left to right, the columns give the Bolocam R.A., Bolocam decl., Bolocam flux density, noise-de-boosted Bolocam flux density, extrapolated flux density at $268 \mathrm{GHz}$ based on our graybody fit to measurements from the three SPIRE bands, and distance from the Bolocam detection to the SPIRE detection. Note that the SPIRE measurements are dominated by confusion noise, which is highly correlated between the bands and is not included in the graybody fit uncertainties. Consequently, the uncertainties on the graybody fits assume that there is either a single source contributing all of the signal in the three SPIRE bands, or that the superposition of sources can be adequately described by a single graybody. For each of these eight candidate sources, the sum of the SPIRE graybody fits is consistent with the de-boosted Bolocam flux density, indicating that the Bolocam candidates are likely robust detections.

Navarro-Frenk-White (gNFW) profile to the common-modesubtraction map, processing the gNFW model through the adaptive-PCA reduction, and subtracting the processed model from the adaptive-PCA map (see Figure 7). The resulting extended-SZ-subtracted adaptive-PCA map contains a total of eight unresolved galaxy candidates with a signal-to-noise $(\mathrm{S} / \mathrm{N})>4$, and we measure the best-fit flux density for each of these candidates after accounting for the filtering effects of the adaptive-PCA reduction (see Table 4). Using these best-fit flux densities and positions, we then process these eight candidates 
through the common-mode reduction and subtract them from the common-mode-subtraction map used for our SZ analysis. In addition, we generate 1000 random realizations of each of the eight candidates based on the measurement uncertainties on the best-fit flux densities, and we add one realization of the uncertainty for each candidate to each of the 1000 noise realizations described in Section 4.1.

In addition to our Bolocam data, we also search for dusty star-forming galaxies in three-band $(250,350$, and $500 \mu \mathrm{m})$ observations obtained with the SPIRE photometer. All of the SPIRE images are dominated by confusion noise from unresolved galaxies (Nguyen et al. 2010), and the effective $\mathrm{rms}$ is $7.2,5.3$, and $5.8 \mathrm{mJy} /$ beam at 250,350 , and $500 \mu \mathrm{m}$, respectively. The SPIRE data are reduced using the Herschel Interactive Processing Environment (Ott et al. 2006; Ott 2010), along with the HerMES SMAP package (Levenson et al. 2010; Viero et al. 2013). Source catalogs are then compiled using the SCAT procedure (Smith et al. 2012), and we identify a total of 200 source candidates within the $14^{\prime} \times 14^{\prime}$ Bolocam coverage with $\mathrm{S} / \mathrm{N}>3$ in any of the SPIRE bands. We note that very few of the SPIRE detections are located within the extended SZ signal detected with Bolocam, and none of the sources located within the extended SZ signal are particularly bright, indicating that there is little contamination of the extended SZ signal due to bright dusty star-forming galaxies.

For each candidate SPIRE galaxy we fit the three-band SPIRE data to a graybody spectral energy distribution of the form

$$
S(v)=A \times v^{1.7} \times B(v, T),
$$

where $B(\nu, T)$ is the Planck blackbody equation and the normalization $A$ and temperature $T$ are free parameters. In performing these fits, we make the assumption that this graybody parameterization describes the emission within the SPIRE PSF regardless of whether the emission is sourced by a single galaxy or many galaxies. Consequently, we do not include the effects of confusion noise in these fits. Using the graybody fits to the SPIRE data, we estimate the $268 \mathrm{GHz}$ flux density centered on each of the 200 SPIRE candidates. We note that, probably due to the fact that multiple sources above the SPIRE measurement noise rms are likely to be present within the extent of the SPIRE PSF, this simple graybody model does not provide an adequate fit to all of the SPIRE source candidates. Specifically, 1/3 of candidates produce a fit PTE $<0.05$, indicating that a graybody fit does not describe the emission detected within the SPIRE PSF for those candidates. In addition, even if we discard that $1 / 3$, the distribution of PTE values for the remaining $2 / 3$ of the candidates is still marginally inconsistent with a uniform distribution, quantified by a KS test PTE of 0.03. This implies that a graybody fit is inadequate to describe a significant fraction of the SPIRE candidates. Unfortunately, it is not practical to fit a more complicated model to the three-band SPIRE data alone due to the lack of spectral information, and so there is no clear model extension to obtain a better fit quality for the SPIRE candidates that are not adequately described using a graybody model. We discuss the implications of this modeling limitation in more detail below.

We find that fourteen of these SPIRE candidates are located within $30^{\prime \prime}$ of the eight Bolocam candidates, indicating that they are possible counterparts. To compare the Bolocam and SPIRE measurements, we first estimate the best-fit noise-de-boosted flux density for the eight candidates detected by Bolocam according to the AzTEC de-boosting algorithm presented in Austermann et al. (2009) by interpolating the tabulated values presented in Downes et al. (2012). As shown in Figure 8 and Table 4, in general we find good agreement between the deboosted Bolocam flux densities and the sum of the extrapolated SPIRE flux densities for all of the likely counterparts. Therefore, the Bolocam candidates are robust detections.

By discarding the 14 SPIRE candidates that are likely counterparts of the Bolocam candidates because they have already been subtracted from the common-mode-subtraction map, along with 24 SPIRE candidates that have an extrapolated $268 \mathrm{GHz}$ $\mathrm{S} / \mathrm{N}<2$, we then generate an image from the remaining 162 SPIRE candidates using the extrapolated $268 \mathrm{GHz}$ SPIRE flux densities convolved with the Bolocam PSF. This image is then processed through the adaptive-PCA reduction, and we subtract it from the corresponding extended-SZ-and-Bolocamcandidate-subtracted adaptive-PCA map (see Figure 7). Removal of this SPIRE template, which we generate independently from our Bolocam data, results in a significant reduction in the rms of the Bolocam image, with $\Delta \chi^{2}=166$. To put this value in context, the total $\chi^{2}$ of the SPIRE template is 240 , and therefore a perfect correlation between the SPIRE extrapolations and the Bolocam data would have resulted in $\Delta \chi^{2}=240$. As a further quality check, we compute the normalization of the SPIRE template that best fits our $268 \mathrm{GHz}$ Bolocam extendedSZ-and-Bolocam-candidate-subtracted adaptive-PCA map and find a value of $0.84 \pm 0.09$ including flux calibration uncertainties. The consistency of this value with unity indicates that the SPIRE template is a good description of the CIB in the $268 \mathrm{GHz}$ Bolocam data.

To subtract these 162 SPIRE-detected sources from our common-mode-subtraction map, we process the SPIRE template through the common-mode reduction and subtract the resulting map from the corresponding common-modesubtraction map we use for SZ analysis. We also generate 1000 map realizations with flux densities for each source randomly distributed according to the uncertainty on the extrapolation and add one such realization to each of the 1000 noise realizations. As described above, the likely Bolocam counterparts are not included in this SPIRE-source template to avoid potential double subtractions, and the $\mathrm{S} / \mathrm{N}<2$ extrapolations are removed to prevent the subtraction of possible false SPIRE detections. We estimate that, between the Bolocam and SPIRE detections, we remove all of the dusty galaxies with a $268 \mathrm{GHz}$ flux density $\gtrsim 1 \mathrm{mJy}$ along with $\simeq 100$ galaxies with lower flux densities, significantly reducing the contamination from these sources on our measurement of the SZ signal (see Figure 9, left).

Due to the fact that the SPIRE candidates are in general not well described by the graybody model, the derived uncertainty on the extrapolation is likely to be underestimated. Therefore, to determine the effect that this underestimation might have on our SZ results, we artificially increase the uncertainties on the SPIRE photometry for each candidate until the reduced $\chi^{2}$ of the graybody fit is equal to 1 . For candidates with a reduced $\chi^{2}<1$, the uncertainties are left unchanged. Although this procedure is unphysical, it does provide a reasonable basis for estimating the uncertainty on the extrapolation for these sources. We find that the difference between the estimated uncertainties on the $268 \mathrm{GHz}$ SZ brightnesses for sub-clusters $\mathrm{B}$ and $\mathrm{C}$ with and without including this artificial increase on the extrapolation uncertainty is $<4 \times 10^{-4} \mathrm{MJy} \mathrm{sr}^{-1}$, or $\lesssim 1 \%$ of the nominal uncertainties. Therefore, we have not accounted for the potentially underestimated uncertainties on the SPIRE candidates that are poorly fit by the graybody model. 

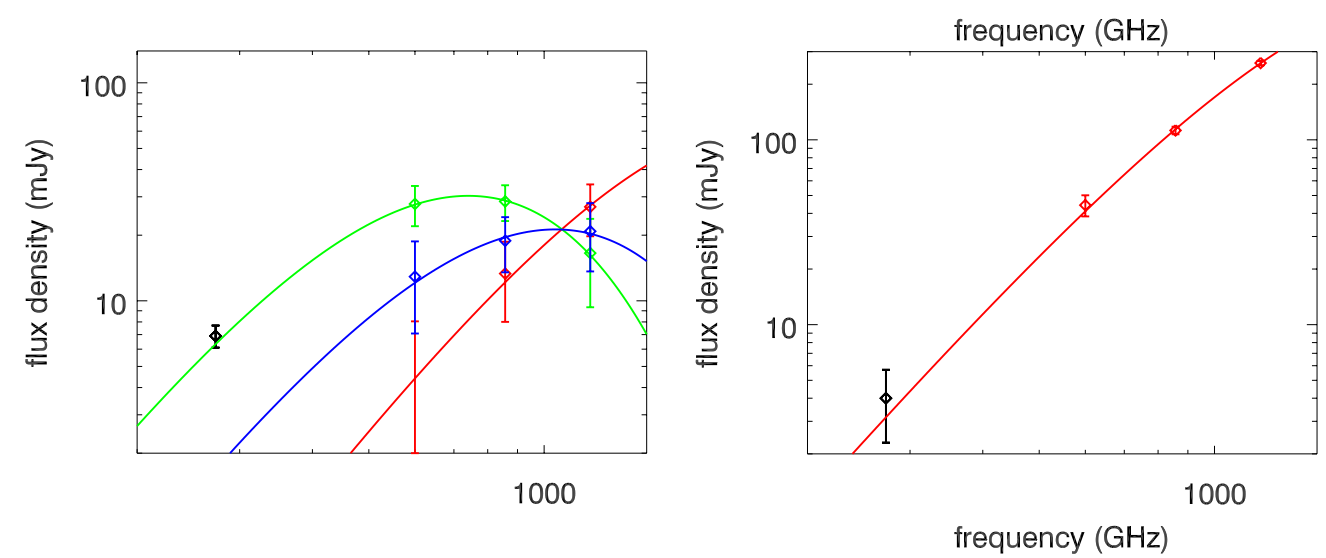

Figure 8. Two of the eight candidate galaxies detected by Bolocam. In each plot the de-boosted Bolocam flux density is shown as a black diamond and the SPIRE flux densities are shown as colored diamonds with a different color for each possible counterpart. The lines show graybody SED fits to the SPIRE data only, with a separate fit for each possible counterpart.

(A color version of this figure is available in the online journal.)
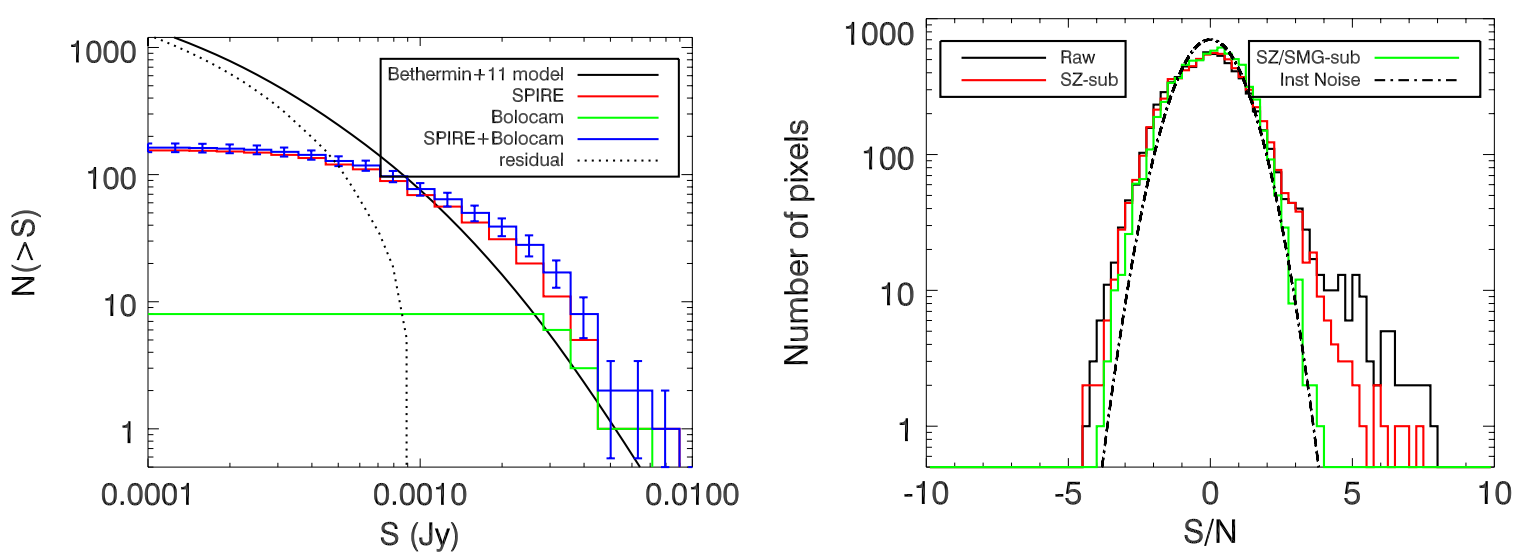

Figure 9. Left: number of galaxies above a given $268 \mathrm{GHz}$ flux density within the $14^{\prime} \times 14^{\prime}$ Bolocam image. The solid black line denotes the Béthermin et al. (2011) model prediction, green denotes the Bolocam detections, red denotes the extrapolated SPIRE detections after removing possible counterparts to the Bolocam detections along with $\mathrm{S} / \mathrm{N}<2$ extrapolations, blue denotes all Bolocam and SPIRE detections, and the dashed black line is the difference between the model and our total detections. Right: S/N histogram for the $268 \mathrm{GHz}$ Bolocam adaptive-PCA map. Black shows the histogram prior to any signal subtraction, red shows the histogram after subtraction of the best-fit extended-SZ model, green shows the histogram after further subtraction of the eight sources detected by Bolocam and the 162 sources detected by SPIRE and extrapolated to $268 \mathrm{GHz}$, and the dot-dashed black line shows the histogram of our jackknife realizations, which contain all of the non-astronomical noise present in our data. The difference in width between the green histogram and the dot-dashed black histogram matches the prediction of the Béthermin et al. (2011) model, indicating that it provides an adequate description of our data.

(A color version of this figure is available in the online journal.)

In addition, we also estimate the uncertainty on the extrapolation due to our particular choice of graybody model. Specifically, we fix the spectral index of the graybody to 1.7 , while the measured spectral indices from large source catalogs vary between $\simeq 1-2$ (e.g., Roseboom et al. 2013). To bound the maximum possible change in the extrapolated CIB template due to this source variation, we rerun our analysis using spectral indices of 1.1 and 2.1 , which correspond to the $1 \sigma$ bounds determined by Roseboom et al. (2013). Using the templates determined from these spectral indices, the $268 \mathrm{GHz} \mathrm{SZ}$ brightnesses toward sub-clusters $\mathrm{B}$ and $\mathrm{C}$ change by $0.001-0.005 \mathrm{MJy} \mathrm{sr}^{-1}$, or by as much as $10 \%$ of their nominal uncertainties. When added in quadrature with our overall uncertainties this amount is negligible, and we therefore have not included it in our uncertainty estimates.

Furthermore, we note that the best-fit amplitude of our nominal CIB template using Bolocam data is $0.84 \pm 0.09$, hinting that the SPIRE data may be producing a template that is slightly over-predicting the CIB at $268 \mathrm{GHz}$. This could be caused by, for example, noise-boosted flux densities in the SPIRE data or an incorrect choice of SED template. To estimate the effects of such a potential bias, we re-estimate the $268 \mathrm{GHz}$ SZ brightnesses of sub-clusters B and C after subtracting an extrapolated SPIRE template with the best-fit Bolocam normalization of 0.84 (rather than 1). Using this renormalized template results in SZ brightnesses that differ by $\lesssim 0.001 \mathrm{MJy} \mathrm{sr}^{-1}$ compared to those found with the default normalization of the template, or by approximately $2 \%$ of our nominal uncertainties. Therefore, the bias associated with a potential over-estimate of the CIB template is negligible, and we have not attempted to account for such a bias in our analysis.

Bolocam and SPIRE do not individually detect the faint galaxies that comprise most of the CIB. Consequently, we use the CIB model determined by Béthermin et al. (2011) to account for the noise fluctuations from these undetected galaxies, as it provides a reasonable estimate for the behavior of the component of the CIB below the SPIRE detection limit. We model the undetected CIB using a number counts distribution obtained by subtracting the number counts already detected by SPIRE and Bolocam from the Béthermin et al. (2011) number counts model. We then generate 1000 random sky realizations of the sources in this population, process each such realization through the 
common-mode reduction, and add one realization of this faint CIB model to each of our 1000 noise realizations.

We note that the Béthermin et al. (2011) model was calibrated using observations of blank sky and therefore is not necessarily an accurate description of the CIB toward a massive cluster like MACS J0717.5+3745. Although the emission from clustermember galaxies at $268 \mathrm{GHz}$ is likely to be negligible compared to the background $\mathrm{CIB},{ }^{18}$ the significant magnification of the background due to gravitational lensing can distort the number counts. In particular, lensing preserves the total surface brightness of the CIB, but causes a significant and spatially dependent change in the number counts (Zemcov et al. 2013). Our data show hints of this change as an excess of sources at bright flux densities, which is consistent with measurements toward massive clusters using AzTEC at the same wavelength (Wardlow et al. 2010; Downes 2009). It is therefore not clear how well the unlensed Béthermin et al. (2011) model describes the faint population of dusty star-forming galaxies toward MACS J0717.5+3745.

To test the validity of the Béthermin et al. (2011) model in describing our MACS J0717.5+3745 data, we add a random sky realization to each jackknife realization of the adaptive-PCA map, where the sky realizations are based on the aforementioned difference between the Béthermin et al. (2011) model and our detected number counts. We find that adding these random sky realizations increases the noise rms by $12.3 \%$. We then fit a Gaussian to the distributions of pixel $\mathrm{S} / \mathrm{N}$ values for the adaptive-PCA map jackknife realizations, and to the actual data after subtraction of the extended-SZ template, the Bolocam detections, and the extrapolated SPIRE detections (see Figure 9, right). We find that the Gaussian standard deviations returned by the fits differ by $11.9 \% \pm 0.8 \%$, in excellent agreement with the prediction based on the Béthermin et al. (2011) model. Therefore, the Béthermin et al. (2011) model provides a good description of the global noise fluctuations due to dim unresolved galaxies at the level we are able to ascertain with our Bolocam data.

In principal, we could subtract some of the signal from these dim unresolved galaxies via, e.g., a cross correlation analysis between the SPIRE and Bolocam maps. Although these dim galaxies are not detected in any single SPIRE band, their signal will be correlated across the ensemble of SPIRE and Bolocam bands. However, the SPIRE data, particularly at $500 \mu \mathrm{m}$, contains a non-negligible amount of diffuse SZ signal. This signal would also be correlated across the multiple bands, and therefore such an analysis could subtract SZ signal from the Bolocam data in addition to CIB signal. Furthermore, the effective reddening of the CIB due to the SZ signal in the SPIRE data could potentially cause a significant over-estimate of the signal when extrapolated to the Bolocam bands. Consequently, to mitigate these effects, we have chosen to subtract only the unresolved bright galaxies individually detected by SPIRE, which should not be significantly contaminated by the diffuse SZ signal. Although beyond the scope of this work, an optimal analysis would jointly constrain a model of the SZ and CIB signals via a simultaneous fit to both the SPIRE and Bolocam data. However, given the relative dimness of the SZ signal in the SPIRE bands, along with the relative dimness of the CIB signal in the Bolocam bands, the improvement from such a joint fit is likely to be minimal compared to our analysis procedure.

\footnotetext{
18 For example, see the arguments presented in Section 4 of Sayers et al. (2013b) based on the results presented in Geach et al. (2006), Bai et al. (2007), Marcillac et al. (2007), Finn et al. (2010), and Rawle et al. (2012).
}

We note that the fluctuations from the CIB are accounted for in our $140 \mathrm{GHz}$ data by adding noise realizations generated according to the power spectrum measurements from SPT (Hall et al. 2010). We do not attempt to subtract any individual galaxies due to the large, and consequently potentially untrustworthy, spectral extrapolation that would be required from the SPIRE measurements. In addition, as noted above, there are very few galaxies detected within the extended region containing bright SZ signal, and none of those galaxies are particularly bright. We note that this is, in general, true for massive clusters, as shown in Zemcov et al. (2013). However, to verify that the CIB is sufficiently dim that we can neglect subtracting it at $140 \mathrm{GHz}$, we extrapolated the SPIRE detections to $140 \mathrm{GHz}$ and removed them from the Bolocam data. Even if this large extrapolation is potentially untrustworthy, it should provide a reasonable estimate of the potential brightness of the CIB at $140 \mathrm{GHz}$. When we subtract this extrapolated CIB, we find that the best-fit $140 \mathrm{GHz}$ SZ brightnesses toward both subcluster B and sub-cluster $\mathrm{C}$ changes by $\simeq 5 \times 10^{-4} \mathrm{MJy} \mathrm{sr}^{-1}$, or by approximately $1 \%$ of the total uncertainty on each subcluster's SZ brightness. Therefore, neglecting to subtract the CIB at $140 \mathrm{GHz}$ has a negligible effect on our overall results.

\section{REFERENCES}

Arnaud, M., Pratt, G. W., Piffaretti, R., et al. 2010, A\&A, 517, A92 Austermann, J. E., Aretxaga, I., Hughes, D. H., et al. 2009, MNRAS, 393, 1573 Bai, L., Marcillac, D., Rieke, G. H., et al. 2007, ApJ, 664, 181 Bennett, C. L., Larson, D., Weiland, J. L., et al. 2013, ApJS, 208, 20 Benson, B. A., Church, S. E., Ade, P. A. R., et al. 2003, ApJ, 592, 674 Béthermin, M., Dole, H., Lagache, G., Le Borgne, D., \& Penin, A. 2011, A\&A, 529, A4

Bhattacharya, S., \& Kosowsky, A. 2008, PhRvD, 77, 083004

Birkinshaw, M. 1999, PhR, 310, 97

Blain, A. W., Smail, I., Ivison, R. J., Kneib, J.-P., \& Frayer, D. T. 2002, PhR, 369,111

Bonafede, A., Feretti, L., Giovannini, G., et al. 2009, A\&A, 503, 707

Bulbul, G. E., Smith, R. K., Foster, A., et al. 2012, ApJ, 747, 32

Carlstrom, J. E., Holder, G. P., \& Reese, E. D. 2002, ARA\&A, 40, 643

Chluba, J., Nagai, D., Sazonov, S., \& Nelson, K. 2012, MNRAS, 426, 510

Conley, A., Guy, J., Sullivan, M., et al. 2011, ApJS, 192, 1

Dorman, B., \& Arnaud, K. A. 2001, in ASP Conf. Ser. 238, Astronomical Data Analysis Software and Systems X, ed. F. R. Harnden, Jr., F. A. Primini, \& H. E. Payne (San Francisco, CA: ASP), 415

Downes, T. P. 2009, PhD thesis, University of Chicago

Downes, T. P., Welch, D., Scott, K. S., et al. 2012, MNRAS, 423, 529

Ebeling, H., Barrett, E., \& Donovan, D. 2004, ApJL, 609, L49

Ebeling, H., Barrett, E., Donovan, D., et al. 2007, ApJL, 661, L33

Ebeling, H., Edge, A. C., \& Henry, J. P. 2001, ApJ, 553, 668

Edge, A. C., Ebeling, H., Bremer, M., et al. 2003, MNRAS, 339, 913

Feldman, H. A., Watkins, R., \& Hudson, M. J. 2010, MNRAS, 407, 2328

Finn, R. A., Desai, V., Rudnick, G., et al. 2010, ApJ, 720, 87

Fruscione, A., McDowell, J. C., Allen, G. E., et al. 2006, Proc. SPIE, 6270, $62701 \mathrm{~V}$

Geach, J. E., Smail, I., Ellis, R. S., et al. 2006, ApJ, 649, 661

Glenn, J., Knowles, B., Rownd, B. K., et al. 2002, in ASP Conf. Ser. 283, A New Era in Cosmology, ed. N. Metcalfe \& T. Shanks (San Francisco, CA: ASP), 398

Griffin, M. J., \& Orton, G. S. 1993, Icar, 105, 537

Haig, D. J., Ade, P. A. R., Aguirre, J. E., et al. 2004, Proc. SPIE, 5498, 78 Hall, N. R., Keisler, R., Knox, L., et al. 2010, ApJ, 718, 632

Hand, N., Addison, G. E., Aubourg, E., et al. 2012, PhRvL, 109, 041101 Hasselfield, M., Moodley, K., Bond, J. R., et al. 2013, arXiv:1303.4714

Hayashi, E., \& White, S. D. M. 2006, MNRAS, 370, L38

Hinshaw, G., Larson, D., Komatsu, E., et al. 2013, ApJS, 208, 19

Holzapfel, W. L., Ade, P. A. R., Church, S. E., et al. 1997, ApJ, 481, 35

Itoh, N., Kohyama, Y., \& Nozawa, S. 1998, ApJ, 502, 7

Itoh, N., \& Nozawa, S. 2004, A\&A, 417, 827

Jauzac, M., Jullo, E., Kneib, J.-P., et al. 2012, MNRAS, 426, 3369

Kitayama, T., Komatsu, E., Ota, N., et al. 2004, PASJ, 56, 17

Kosowsky, A., \& Bhattacharya, S. 2009, PhRvD, 80, 062003

Kuntz, K. D., \& Snowden, S. L. 2008, ApJ, 674, 209 
Lee, J., \& Komatsu, E. 2010, ApJ, 718, 60

Levenson, L., Marsden, G., Zemcov, M., et al. 2010, MNRAS, 409, 83

Li, C. K., Jia, S. M., Chen, Y., et al. 2012, A\&A, 545, A100

Limousin, M., Ebeling, H., Richard, J., et al. 2012, A\&A, 544, A71

Ma, C.-J., Ebeling, H., \& Barrett, E. 2009, ApJL, 693, L56

Ma, Y.-Z., Branchini, E., \& Scott, D. 2012, MNRAS, 425, 2880

Mahdavi, A., Hoekstra, H., Babul, A., et al. 2013, ApJ, 767, 116

Mak, D. S. Y., Pierpaoli, E., \& Osborne, S. J. 2011, ApJ, 736, 116

Mantz, A., Allen, S. W., Ebeling, H., Rapetti, D., \& Drlica-Wagner, A. 2010, MNRAS, 406, 1773

Marcillac, D., Rigby, J. R., Rieke, G. H., \& Kelly, D. M. 2007, ApJ, 654, 825

Markwardt, C. B. 2009, in ASP Conf. Ser. 411, Astronomical Data Analysis Software and Systems XVIII, ed. D. A. Bohlender, D. Durand, \& P. Dowler (San Francisco, CA: ASP), 251

Mauskopf, P. D., Horner, P. F., Aguirre, J., et al. 2012, MNRAS, 421, 224

Medezinski, E., Umetsu, K., Nonino, M., et al. 2013, ApJ, 777, 43

Meneghetti, M., Fedeli, C., Zitrin, A., et al. 2011, A\&A, 530, A17

Mroczkowski, T., Dicker, S., Sayers, J., et al. 2012, ApJ, 761, 47

Nevalainen, J., David, L., \& Guainazzi, M. 2010, A\&A, 523, A22

Nguyen, H. T., Schulz, B., Levenson, L., et al. 2010, A\&A, 518, L5

Nozawa, S., Itoh, N., \& Kohyama, Y. 1998a, ApJ, 508, 17

Nozawa, S., Itoh, N., \& Kohyama, Y. 1998b, ApJ, 507, 530

Nozawa, S., Itoh, N., Suda, Y., \& Ohhata, Y. 2006, NCimB, 121, 487

Nusser, A., \& Davis, M. 2011, ApJ, 736, 93

Osborne, S. J., Mak, D. S. Y., Church, S. E., \& Pierpaoli, E. 2011, ApJ, 737, 98

Ott, S. 2010, in ASP Conf. Ser. 434, Astronomical Data Analysis Software and Systems XIX, ed. Y. Mizumoto, K.-I. Morita, \& M. Ohishi (San Francisco, CA: ASP), 139

Ott, S., Bakker, J., Brumfitt, J., et al. 2006, in ASP Conf. Ser. 351, Astronomical Data Analysis Software and Systems XV, ed. C. Gabriel, C. Arviset, D. Ponz, \& S. Enrique (San Francisco, CA: ASP), 516

Planck Collaboration, Ade, P. A. R., Aghanim, N., et al. 2013a, arXiv:1303.5069 Planck Collaboration, Ade, P. A. R., Aghanim, N., et al. 2013b, arXiv: 1303.5075 Planck Collaboration, Ade, P. A. R., Aghanim, N., et al. 2013c, arXiv:1303.5076 Planck Collaboration, Ade, P. A. R., Aghanim, N., et al. 2013d, arXiv: 1303.5090 Prokhorov, D. A., \& Colafrancesco, S. 2012, MNRAS, 424, L49
Rawle, T. D., Edge, A. C., Egami, E., et al. 2012, ApJ, 747, 29

Reese, E. D., Kawahara, H., Kitayama, T., et al. 2010, ApJ, 721, 653

Reichardt, C. L., Shaw, L., Zahn, O., et al. 2012, ApJ, 755, 70

Rephaeli, Y. 1995a, ARA\&A, 33, 541

Rephaeli, Y. 1995b, ApJ, 445, 33

Roseboom, I. G., Dunlop, J. S., Cirasuolo, M., et al. 2013, arXiv:1308.4443

Ruan, J. J., Quinn, T. R., \& Babul, A. 2013, MNRAS, 432, 3508

Sanders, J. S. 2006, MNRAS, 371, 829

Sayers, J., Czakon, N. G., \& Golwala, S. R. 2012, ApJ, 744, 169

Sayers, J., Czakon, N. G., Mantz, A., et al. 2013a, ApJ, 768, 177

Sayers, J., Golwala, S. R., Ameglio, S., \& Pierpaoli, E. 2011, ApJ, 728, 39

Sayers, J., Mroczkowski, T., Czakon, N. G., et al. 2013b, ApJ, 764, 152

Sazonov, S. Y., \& Sunyaev, R. A. 1998, ApJ, 508, 1

Smith, A. J., Wang, L., Oliver, S. J., et al. 2012, MNRAS, 419, 377

Smith, R. K., Brickhouse, N. S., Liedahl, D. A., \& Raymond, J. C. 2001, ApJL, 556, L91

Snowden, S. L., Mushotzky, R. F., Kuntz, K. D., \& Davis, D. S. 2008, A\&A, 478,615

Story, K. T., Reichardt, C. L., Hou, Z., et al. 2012, arXiv:1210.7231

Sunyaev, R. A., \& Zel'dovich, Y. B. 1972, CoASP, 4, 173

Suzuki, N., Rubin, D., Lidman, C., et al. 2012, ApJ, 746, 85

Thompson, R., \& Nagamine, K. 2012, MNRAS, 419, 3560

Tully, R. B., \& Fisher, J. R. 1977, A\&A, 54, 661

van Weeren, R. J., Röttgering, H. J. A., Brüggen, M., \& Cohen, A. 2009, A\&A, 505,991

Viero, M. P., Wang, L., Zemcov, M., et al. 2013, ApJ, 772, 77

Waizmann, J.-C., Redlich, M., \& Bartelmann, M. 2012, A\&A, 547, A67

Wardlow, J. L., Smail, I., Wilson, G. W., et al. 2010, MNRAS, 401, 2299

Weiland, J. L., Odegard, N., Hill, R. S., et al. 2011, ApJS, 192, 19

Wu, J., Tsai, C.-W., Sayers, J., et al. 2012, ApJ, 756, 96

Zahn, O., Reichardt, C. L., Shaw, L., et al. 2012, ApJ, 756, 65

Zemcov, M., Aguirre, J., Bock, J., et al. 2012, ApJ, 749, 114

Zemcov, M., Blain, A., Cooray, A., et al. 2013, ApJL, 769, L31

Zhuravleva, I., Churazov, E., Kravtsov, A., et al. 2013, MNRAS, 428,3274

Zitrin, A., Broadhurst, T., Rephaeli, Y., \& Sadeh, S. 2009, ApJL, 707, L102 